\title{
The age, life expectancy, and space density of Post Common Envelope Binaries
}

\author{
M. R. Schreiber ${ }^{1}$ and B. T. Gänsicke ${ }^{2}$ \\ 1 Université Louis Pasteur, Observatoire de Strasbourg, 11 rue de l'Université, 67000 Strasbourg, France \\ ${ }^{2}$ Department of Physics and Astronomy, University of Southampton, Highfield, Southampton SO17 1BJ, UK
}

Received 29 November 2002 / Accepted 26 May 2003

\begin{abstract}
We present a sample of 30 well observed Post Common Envelope Binaries (PCEBs). Deriving the cooling age of the white dwarfs, we show that the PCEB population is dominated by young systems. Having calculated the orbital evolution of the systems under the assumption of two different prescriptions for the angular momentum loss, we find that most of the systems have not yet completed a significant fraction of their PCEB life time. We therefore predict the existence of a large population of old PCEBs containing cold white dwarfs $\left(T_{\text {eff }} \lesssim 15000 \mathrm{~K}\right)$. Our calculations show that nearly half of the PCEBs in our sample will evolve into a semi-detached configuration and start mass transfer in less than the Hubble-time. These systems are thus representative for progenitors of the current CV population. Only one of them (V471 Tau) will evolve into a long-period $\left(P_{\text {orb }} \gtrsim 4 \mathrm{~h}\right) \mathrm{CV}$, and a significant fraction of the systems will start mass transfer in the period gap. Having estimated the distances of the PCEBs in the sample, we derive a space density of $\rho_{\mathrm{PCEB}} \sim 6-30 \times 10^{-6} \mathrm{pc}^{-3}$, depending on the assumed angular momentum loss prescription. Taking into account the evolutionary time scales we compute a lower limit for the CV space density, predicted by the currently known PCEB population of $\rho_{\mathrm{CV}} \gtrsim 10^{-5} \mathrm{pc}^{-3}$. Finally, we discuss possible observational selection effects and conclude that the observed PCEB population is probably highly incomplete.
\end{abstract}

Key words. accretion, accretion discs - stars: binaries: close - stars: novae, cataclysmic variables

\section{Introduction}

In cataclysmic variables (CVs) (Warner 1995, for an encyclopaedic review) a white dwarf accretes material from its Roche-lobe filling secondary, typically a K- or M-dwarf.

The standard picture for the formation of CVs assumes that the progenitor systems were moderately wide binaries consisting of an intermediate mass star with a low mass companion. Once the more massive star evolves to giant dimensions and fills its Roche-lobe, runaway mass transfer onto the less massive star starts and the systems enters into a Common Envelope phase (CE). Friction within the envelope extracts angular momentum, which tightens the orbit. Post common envelope binaries (PCEB) with orbital periods of a few days or less may evolve into a semi-detached CV configuration through orbital angular momentum loss. Plausible angular momentum loss agencies are gravitational radiation and - much more efficient magnetic braking.

The standard paradigm describing the evolution of CVs after the onset of mass transfer is known as disrupted magnetic braking (Rappaport et al. 1983; Paczynski \& Sienkiewicz 1983; Spruit \& Ritter 1983; see King 1988 for a review). In brief, the concept of this theory is that the evolution of CVs is divided into two main phases, depending on the prevailing

Send offprint requests to: M. R. Schreiber, e-mail: mschrei@astro.u-strasbg.fr angular momentum loss mechanism. Stellar magnetic braking dominates in CVs whose Roche-lobe filling donor stars still have a radiative core, which is the case for orbital periods $P_{\text {orb }} \geq 3 \mathrm{~h}$. Once that the donor stars become fully convective at $P_{\text {orb }} \simeq 3 \mathrm{~h}$, magnetic braking ceases. For $P_{\text {orb }}<3 \mathrm{~h}$ gravitational radiation takes over as a much less efficient angular momentum loss mechanism, resulting in longer evolution time scales. As a consequence of the high mass loss rate in the magnetic braking regime, the donor stars in CVs with $P_{\text {orb }}>3 \mathrm{~h}$ are somewhat expanded. The mass loss rate decreases when magnetic braking ceases at $P_{\text {orb }} \simeq 3 \mathrm{~h}$, and the donor star reacts by relaxing to its thermal equilibrium configuration with a radius that is smaller than its Roche-lobe radius. As a result, the mass transfer shuts off completely, and the CV becomes an inactive detached white dwarf/dM binary that evolves towards shorter periods through emission of gravitational radiation. At $P_{\text {orb }} \simeq 2 \mathrm{~h}$, the secondary fills again its Roche volume and restarts the mass transfer (though at a much lower rate than in the long-period CVs).

The main merit of the disrupted magnetic braking model is that it can successfully explain the period gap, i.e. the statistically significant paucity of known CVs with orbital periods in the range $2-3 \mathrm{~h}$. However, a number of predictions of this standard model are in strong disagreement with the observations: (1) the predicted minimum orbital period is $65-70 \mathrm{~min}$, $10 \%$ shorter than the observed value (Kolb \& Baraffe 1999); 
(2) CVs should spend most of their lifetime near the minimum period, increasing the discovery probability for such systems. Population syntheses predict a significant accumulation of systems near the minimum period, which is not observed (Kolb \& Baraffe 1999); (3) whereas all population syntheses predict that $\sim 95 \%$ of the entire CV population should have orbital periods $<2 \mathrm{~h}$ (e.g. Howell et al. 1997), similar numbers of CVs are observed above and below the period gap; (4) while the population models predict a space density of a few $10^{-5}$ to a few $10^{-4} \mathrm{pc}^{-3}$ (de Kool 1992; Politano 1996), the space density derived from the currently known sample of CVs is only several few $10^{-6} \mathrm{pc}^{-3}$ (e.g. Downes 1986; Ringwald 1996). If the population models are correct, we have identified so far only a small fraction $(\sim 1-10 \%)$ of the existing $\mathrm{CV}$ population (Gänsicke et al. 2002); and finally (5) there is no observational evidence for a discontinuous change in the spin-down rate due to magnetic braking between late-type field stars that are fully convective and those that have a radiative core.

It is apparent that detailed populations studies of the $\mathrm{CV}$ progenitors (i.e. PCEBs) are extremely important for a global understanding of CV formation and evolution, and extensive theoretical analyses of these systems have been performed in the past (Ritter 1986; de Kool 1992; de Kool \& Ritter 1993; King et al. 1994; Politano 1996). However, the relatively small number of known PCEBs limited so far the comparison of these studies with observations. Throughout the last decade, a number of additional PCEBs have been discovered, significantly improving the statistical properties of the known PCEB population.

In this paper, we analyse the properties of a sample of well-observed PCEBs and discuss possible implications for the PCEB/CV evolution. In Sect. 2, we briefly summarise recent alternatives/additions to the standard CV evolution theory outlined in the Introduction. The different angular momentum loss prescription that have been used in the context of CV evolution are described in Sect.3. We introduce our sample of PCEBs in Sect. 4, and discuss the past and future evolution of these stars in Sects. 5 and 6, respectively. Section 7 provides the distances to the PCEBs in our sample. In Sect. 8, we compute the space density of PCEBs, and use this result to estimate the space density of the present-day CV population. The effects of observational biases are discussed in Sect. 9. Finally, Sect. 10 summarises our findings.

When discussing the properties of the present-day PCEB population in the context of the present-day $\mathrm{CV}$ population, specifically in Sects. 6 and 8, we will use the term precataclysmic variables (pre-CVs) to denote those systems which can be regarded as representative for the progenitors of the current $\mathrm{CV}$ population - i.e. PCEBs that evolve into a semidetached configuration in less than the Hubble-time ${ }^{1}$.

\footnotetext{
${ }^{1}$ Obviously also PCEBs with evolutionary time scales longer than the Hubble-time will form CVs, but they differ from the progenitors of present-day CVs. This difference is the only reason for our definition of "pre-CVs".
}

\section{Recent modifications of the "standard scenario" of CV formation and evolution}

The "standard scenario" of CV formation and evolution outlined in the introduction has essentially remained unchanged during the last two decades, even though being challenged with a number of alternative suggestions (Livio \& Pringle 1994; King \& Kolb 1995; Clemens et al. 1998; Kolb et al. 1998). Very recently, two far-reaching modifications for the standard scenario have been proposed.

Sills et al. (2000) and Pinsonneault et al. (2002) presented theoretical models of the angular momentum evolution of low mass stars $\left(0.1-0.5 M_{\odot}\right)$ and compared their models to rotational data from open clusters of different ages to infer the rotational history of low-mass stars and the dependence of initial conditions and rotational evolution on mass. The studies of Sills et al. (2000), Pinsonneault et al. (2002), and Andronov et al. (2003) have two important consequences for the theory of $\mathrm{CV}$ evolution. On one hand, angular momentum loss via magnetic braking is less efficient above the fully convective boundary than in the standard CV model, and as a result the evolution time scale of PCEBs containing a secondary with a radiative core is expected to be 2 orders of magnitude longer than in the standard model. On the other hand, the observed angular momentum loss properties show no evidence for a change in behaviour at the fully convective boundary. Magnetic braking remains, hence, an important angular momentum loss mechanism in PCEBs with a fully convective secondary, and these systems evolve faster than in the standard scenario. Due to the increased angular momentum loss below the gap, the theoretical orbital minimum increases, which is in better agreement with the observations (Kolb \& Baraffe 1999; Patterson 1998; King et al. 2002).

However, the evolution time scale of CVs above the gap is much longer than in the standard scenario, and the existence of the observed period gap is tentatively explained by Pinsonneault et al. (2002) by two separated populations of CVs with differently evolved donor stars. One problem with this modification of the standard CV evolution scenario is that the reduced angular momentum loss above the period gap predicts mass transfer rates that are significantly lower than the values derived from the observations. This issue is discussed in more detail in Sect. 3.

King \& Schenker (2002) and Schenker \& King (2002) postulate that the PCEB evolution into a semi-detached configuration takes much longer than in the standard scenario. Without modifying the standard angular momentum loss prescription, such a situation would arise if the frictional angular momentum loss during the CE is less efficient, the PCEBs exit the CE with wider orbits, and evolve into the semi-detached CV configuration only on longer time scales than typically assumed. If the time scale to initiate mass transfer is of the order of the galactic age $\left(\sim 10^{10} \mathrm{yrs}\right)$, both the minimum orbital period problem and the space density problem are solved as a much smaller number of CVs has been produced to date compared to the standard scenario, and the present-day CV population has not yet reached the minimum period. Finally King \& Schenker (2002) speculate that the longer evolution time scale for PCEBs proposed in 
their model might be related to a reduced magnetic braking as suggested by Andronov et al. (2003). However, if the assumed birth rate of the PCEB progenitor binaries remains unchanged, a longer PCEB phase must result in a very large population of old PCEBs. In the following sections, we analyse the properties of the known PCEB sample and discuss the results in the framework of both the standard CV evolution and the context of a revised magnetic braking prescription.

\section{Angular momentum loss}

Angular momentum loss (AML) drives the evolution of the binaries and hence, our understanding of AML is the main ingredient of CV and PCEB evolution. It is believed that PCEBs as well as CVs lose angular momentum via gravitational radiation and magnetic braking.

The rate of AML due to radiation of gravitational waves from Einstein's quadrupole formula is

$\dot{J}_{\mathrm{GR}}=-\frac{32 G^{7 / 3}}{5 c^{5}} \frac{M_{1}{ }^{2} M_{\mathrm{sec}}{ }^{2}}{\left(M_{1}+M_{\mathrm{sec}}\right)^{2 / 3}}\left(\frac{2 \pi}{P_{\mathrm{orb}}}\right)^{7 / 3}$,

with $M_{1}$ and $M_{\text {sec }}$ the mass of the primary and the secondary.

Unfortunately, the efficiency of magnetic braking is rather uncertain (see the Introduction and Sect. 2) and it may have been overestimated in the past (see Pinsonneault et al. 2002). In the following analysis of the currently known PCEB sample we use, therefore, two different prescriptions of AML to calculate the evolution of our sample of PCEBs.

(1) Disrupted magnetic braking until the secondary becomes fully convective and magnetic braking ceases (following the standard scenario of Verbunt \& Zwaan 1981; Rappaport et al. 1983). In this case, the AML is given by

$\dot{J}_{\mathrm{VZ}}=-3.8 \times 10^{-30} M_{\mathrm{sec}} R_{\odot}{ }^{4}\left(\frac{R_{\mathrm{sec}}}{R_{\odot}}\right)^{\gamma}\left(\frac{2 \pi}{P_{\mathrm{orb}}}\right)^{3}$.

The original version of the magnetic braking law corresponds to $\gamma=4.0$ (Verbunt \& Zwaan 1981). In the context of the $\mathrm{CV}$ evolution $\gamma=2.0$ is frequently used, and hence we adopt this value throughout this work.

(2) The empirical AML prescription derived from open cluster data of single stars is (Sills et al. 2000),

$\dot{J}_{\mathrm{SPT}}= \begin{cases}-K_{\mathrm{W}}\left(\frac{R_{\mathrm{sec}} M_{\odot}}{M_{\mathrm{sec}} R_{\odot}}\right)^{0.5} \omega^{3} & \text { for } \omega \leq \omega_{\text {crit }} \\ -K_{\mathrm{W}}\left(\frac{R_{\mathrm{sec}} M_{\odot}}{M_{\mathrm{sec}} R_{\odot}}\right)^{0.5} \omega \omega_{\text {crit }}^{2} & \text { for } \omega>\omega_{\text {crit }},\end{cases}$

where $\omega_{\text {crit }}$ depends on the mass of the secondary. For stars with masses $M \gtrsim M_{\odot}$ a Rossby scaling matches the observations

$\omega_{\text {crit }}=\omega_{\text {crit } \odot} \frac{\tau_{\odot}}{\tau\left(M_{\text {sec }}\right)}$,

with $\tau$ being the convective overturn time (Krishnamurthi et al. 1997). For lower masses the dependence of $\omega_{\text {crit }}$ on mass is found to be stronger than given by Eq. (4) (Sills et al. 2000). Throughout this work we adopted the values for $\omega_{\text {crit }}$ given by Andronov et al. (2003). Notice, for the systems in our sample we find $\omega=10-10^{2} \times \omega_{\text {crit }}$.

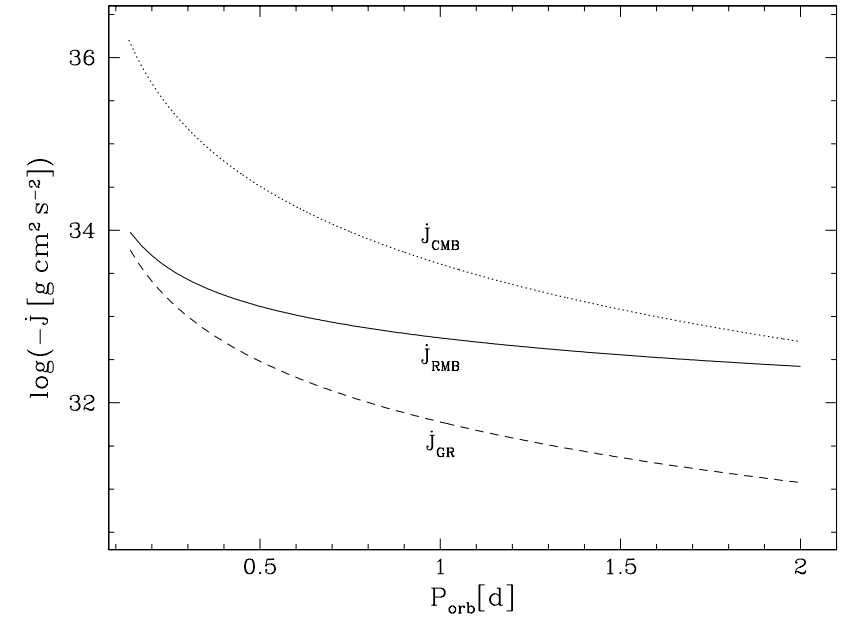

Fig. 1. Angular momentum loss in PCEBs as a function of the orbital period. We assumed $M_{\mathrm{sec}}=0.4 M_{\odot}$ and $M_{1}=0.6 M_{\odot}$. Apparently the revised magnetic braking prescriptions is of similar efficiency as the classical prescription for long orbital periods whereas it is only a few times the angular momentum loss due to gravitational radiation for short orbital periods.

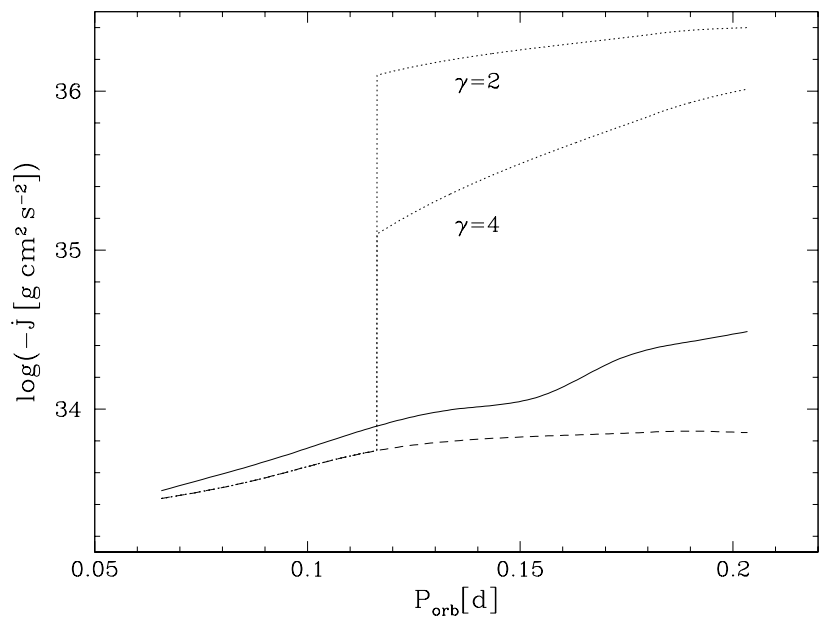

Fig. 2. Angular momentum loss in CVs. As in Fig. 1 we used $M_{1}=$ $0.6 M_{\odot}$ (see also Pinsonneault et al. 2002; Andronov et al. 2003). The dotted lines correspond to Eq. (5) with two values for $\gamma$ (see Eq. (2)). The abrupt decrease displays the shutoff of magnetic braking for $M_{\mathrm{sec}}<0.3 M_{\odot}$. The dashed line represents angular momentum loss by gravitational radiation only whereas the solid line refers to the revised angular momentum loss prescription (Eq. (6)).

In the following sections we distinguish between the two currently discussed AML prescriptions using the notation $\dot{J}_{\mathrm{CMB}}$ and $\dot{J}_{\mathrm{RMB}}$ when referring to the classical respectively the reduced AML prescription:

$\dot{J}_{\mathrm{CMB}}=\left\{\begin{array}{rll}\dot{J}_{\mathrm{VZ}}+\dot{J}_{\mathrm{GR}} & \text { for } & M_{\mathrm{sec}}>0.3 M_{\odot} \\ \dot{J}_{\mathrm{GR}} & \text { for } & M_{\mathrm{sec}} \leq 0.3 M_{\odot}\end{array}\right.$

and

$\dot{J}_{\mathrm{RMB}}=\dot{J}_{\mathrm{SPT}}+\dot{J}_{\mathrm{GR}}$.

Figure 1 shows the AML as a function of $P_{\text {orb }}$ for PCEBs. Figure 2 compares the angular momentum loss agencies 
$\dot{J}_{\mathrm{CMB}}, \dot{J}_{\mathrm{GR}}$, and $\dot{J}_{\mathrm{RMB}}$ for CVs (see also Pinsonneault et al. 2002; Andronov et al. 2003).

It is worth noting that the reduced magnetic braking prescription is not only subject to observational uncertainties (as stated by Andronov et al. 2003 themselves) but also unable to explain the mass accretion rates derived from observations for CVs above the orbital period gap (e.g. Patterson 1984). This is a firm conclusion unless (1) all nova-like systems represent short-lived high accretion states or (2) there exists an additional AML mechanism in these systems. Referring to (1) we note the possibility of irradiation induced mass transfer cycles (e.g. King et al. 1995) and that nova eruptions widen the mass transfer spectrum (Kolb 2002). Concerning (2) we note that circumbinary disks in CVs have been suggested as rather efficient additional AML agents (Spruit \& Taam 2001; Dubus et al. 2002). Thus, while the reduced AML prescription has a problem to explain the "observed" mass transfer rates of CVs above the gap, it is currently not possible to rule out its validity because of the additional complications just mentioned ${ }^{2}$.

Considering the described uncertainties of the AML in close binaries, we discuss the current PCEB population in the context of both prescriptions, $\dot{J}_{\mathrm{CMB}}$ and $\dot{J}_{\mathrm{RMB}}$.

\section{PCEBs: The present}

We have compiled for our present study a sample of PCEBs with well-established characteristics from various literature sources, including the catalogues of Ritter \& Kolb (1998) and Kube et al. (2002). We exclude from our sample systems with $M_{\text {sec }}>M_{1}$ (such as V651 Mon), systems with $P_{\text {orb }} \gtrsim 2 \mathrm{~d}$ (such as Feige 24), and systems with sub-giant secondary stars (such as FF Aqr). Table 1 lists the properties of our PCEB sample, which consists of 18 systems containing a white dwarf primary and 12 systems containing an sdOB primary (of which four are the central stars of planetary nebulae). These 30 systems represent the currently known population of well-observed PCEBs which will evolve eventually into a semi-detached configuration and turn into a $\mathrm{CV}$ and for which reliable observational data exists.

It is interesting to note that the radii of the secondaries derived from observations (Table 1) are generally in good agreement with the ZAMS mass-radius relation given by Politano (1996). Exceptions are the secondary stars in the binary planetary nebulae BE UMa, KV Vel, V477 Lyr, and UU Sge whose radii exceed the ZAMS value by a factor of $\sim 2$ (Fig. 3 ). This effect can be explained by accretion of matter onto the secondary at a relatively high rate during the recent CE-phase. Numerical calculations of accreting low-mass stars have been performed by Prialnik \& Livio (1985) for a fully convective star of $M=0.3 M_{\odot}$ and Fujimoto \& Iben (1989) for a low mass main sequence star of $M=0.75 M_{\odot}$. The calculations show that rather recent accretion at a rate of $\sim 10^{-5} M_{\odot} \mathrm{yrs}^{-1}$ to $\sim 10^{-7} M_{\odot} \mathrm{yrs}^{-1}$ can explain the observed expansion of the

\footnotetext{
${ }^{2}$ In this context we note that it might be easier to test our understanding of AML using a large (not yet identified) sample of PCEBs, as all the complications related to mass transfer are not present in these binaries.
}

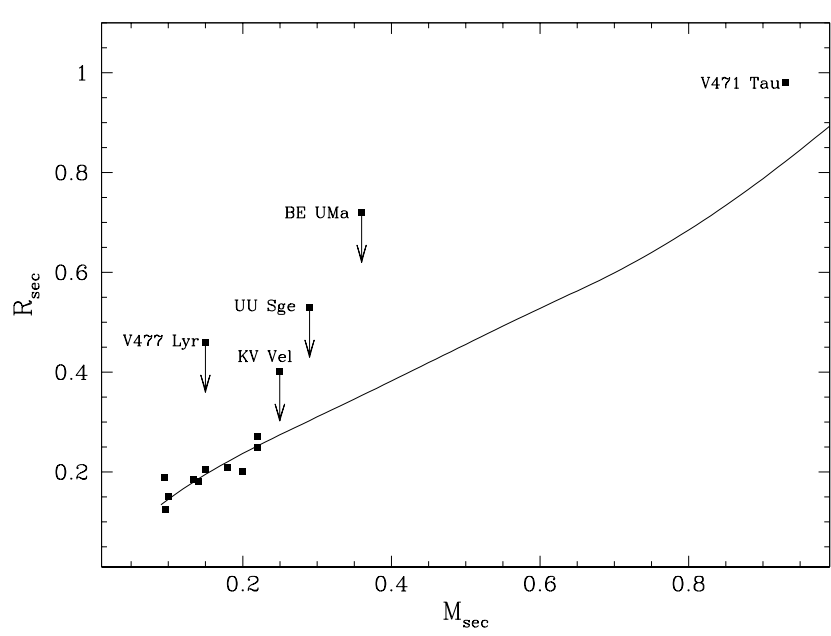

Fig. 3. The ZAMS mass radius relation given by Politano (1996) and the positions of the PCEB secondaries for which an observationally determined radius is available. The arrows indicate that the radii of the secondaries in these planetary nebulae are larger due to recent accretion onto the secondary during a $\mathrm{CE}$ phase. Before becoming a CV the secondary will have contracted to its main-sequence radius.

secondary. As the time scale on which the secondary contracts is shorter than the binary evolution time scale we use radii calculated from the mass-radius relation of Politano (1996) for the four planetary nebulae in our calculations (Sects. 5, and 6).

A peculiar system is the Hyades PCEB V471 Tau, located in the upper right corner of Fig. 3. Apparently, the secondary is expanded with respect to the ZAMS mass radius relation. Using radiometric measurements and ground based Doppler imaging O'Brien et al. (2001) explain the oversized secondary in V471 Tau as the result of a large fraction of the secondaries surface being covered by star spots, lowering its average effective temperature. In order to maintain the luminosity of a $0.93 M_{\odot}$ dwarf, the radius of the star has, hence, to exceed the ZAMS value. It is worth to note that also the white dwarf in V471 Tau represents an evolutionary paradox as it is the hottest (and therefore youngest) as well as the most massive white dwarf in the Hyades cluster. O'Brien et al. (2001) discuss the possibility that V471 Tau is descended from a blue straggler, which would imply that its progenitor was initially a triple system. Nevertheless, despite its curious nature, V471 Tau is the best-observed PCEB, and, as described in detail in Sect. 6, it will be the PCEB from our sample that starts mass transfer at the longest orbital period.

Final notes concern MT Ser, UUSge, EC 13471-1258, BPM 71214, PG 1017-086 and AADor. The orbital period of MTSer might be as twice as long as given in Table 1, and the system may consist of two hot subdwarfs rather than a subdwarf plus cool companion (Bruch et al. 2001). The large discrepancy between the two values for the temperatures of the primary in UU Sge given in Table 1 results from the fact that Bell et al. (1994) could fit the observed light curve equally well by assuming either limb-darkening or limbbrightening. The given values may therefore be interpreted as upper and lower limits. The binary parameter for EC 134711258 and BPM 71214 given in Table 1 indicate that these 
Table 1. Sample of PCEBs with orbital periods $P \lesssim 2$ days, $M_{1}>M_{\mathrm{sec}}$ and a main-sequence secondary star.

\begin{tabular}{|c|c|c|c|c|c|c|c|c|}
\hline Object & alt. name & Sp1 & $P_{\text {orb }}[\mathrm{d}]$ & $M_{1}\left[M_{\odot}\right]$ & $M_{\mathrm{sec}}\left[M_{\odot}\right]$ & $R_{\mathrm{sec}}\left[R_{\odot}\right]$ & $T_{\text {eff }}[\mathrm{K}]$ & Ref. \\
\hline RR Cae & LFT 349 & WD & 0.304 & 0.467 & 0.095 & 0.189 & 7000 & 1,2 \\
\hline EG UMa & Case 1 & WD & 0.668 & $0.64 \pm 0.03$ & $0.42 \pm 0.04$ & $0.40 \%$ & $13125 \pm 125$ & 3 \\
\hline EC 13471-1258 & & WD & 0.151 & $0.77 \pm 0.04$ & $0.58 \pm 0.05$ & 0.42 & $14085 \pm 100$ & 4 \\
\hline BPM 71214 & & WD & 0.201 & $0.77 \pm 0.06$ & 0.4 & $0.38 \%$ & $17200 \pm 1000$ & 4 \\
\hline HR Cam & GD 448 & WD & 0.103 & $0.41 \pm 0.01$ & $0.096 \pm 0.004$ & $0.125 \pm 0.020$ & 19000 & 5,6 \\
\hline UZSex & PG 1026+002 & WD & 0.597 & $0.65 \pm 0.23$ & $0.22 \pm 0.05$ & $0.25 \pm 0.03$ & $\begin{array}{l}17600 \pm 2000 \\
19900 \pm 330\end{array}$ & $\begin{array}{l}7 \\
8\end{array}$ \\
\hline ВРМ 6502 & LTT 3943 & WD & 0.337 & $0.5 \pm 0.05$ & $0.16 \pm 0.09$ & $0.204^{\%}$ & $\begin{array}{l}20640 \pm 200 \\
21380 \pm 258 \\
20311 \pm 532\end{array}$ & $\begin{array}{l}9 \\
2 \\
10\end{array}$ \\
\hline $\mathrm{HZ} 9$ & & WD & 0.564 & $0.51 \pm 0.1$ & $0.28 \pm 0.04$ & $0.296^{\%}$ & $\begin{array}{l}20000 \pm 2000 \\
17400\end{array}$ & $\begin{array}{l}11,12,13 \\
\text { this work }\end{array}$ \\
\hline MS Peg & GD 245 & WD & 0.174 & $0.48 \pm 0.02$ & $0.22 \pm 0.02$ & $0.27 \pm 0.02$ & 22170 & 14 \\
\hline CCCet & PG 0308+096 & WD & 0.284 & $0.39 \pm 0.1$ & $0.18 \pm 0.05$ & $0.21 \pm 0.02$ & $26200 \pm 2000$ & 7 \\
\hline HW Vir & $\mathrm{BD}-07^{\circ} 4377$ & $\mathrm{sdB}$ & 0.117 & $0.48 \pm 0.09$ & $0.14 \pm 0.02$ & $0.180 \pm 0.011$ & $28488 \pm 208$ & 15 \\
\hline HS 0705+6700 & & $\mathrm{sdB}$ & 0.096 & 0.483 & 0.134 & 0.186 & 28800 & 16 \\
\hline LM Com & Ton $617^{\dagger}$ & WD & 0.259 & $0.45 \pm 0.05$ & $0.28 \pm 0.05$ & $0.296^{\%}$ & 29300 & 17 \\
\hline PG 1017-086 & & $\mathrm{sdB}$ & 0.073 & 0.5 & $0.078_{-0.006}^{+0.005}$ & $0.085 \pm 0.04$ & 30300 & 18 \\
\hline V471 Tau & $\mathrm{BD}+16^{\circ} 516$ & WD & 0.521 & $0.84 \pm 0.05$ & $0.93 \pm 0.07$ & $0.98 \pm 0.10$ & $34500 \pm 1000$ & 19 \\
\hline NY Vir & PG 1336-018 & $\mathrm{sdB}$ & 0.101 & 0.5 & 0.15 & $0.205 \pm 0.01$ & $33000 \pm 3000$ & 20 \\
\hline AA Dor & LB 3459 & $\mathrm{sdO}$ & 0.262 & $0.330 \pm 0.003$ & $0.066 \pm 0.001$ & $0.10 \pm 0.01^{!}$ & $42000 \pm 1000$ & 21 \\
\hline RE $2013+400$ & & WD & 0.706 & $0.56 \pm 0.03$ & $0.18 \pm 0.04$ & $0.221^{\%}$ & $\begin{array}{l}49000 \pm 700 \\
47800 \pm 2400\end{array}$ & $\begin{array}{l}22 \\
23\end{array}$ \\
\hline GK Vir & PG $1413+015$ & WD & 0.344 & $0.51 \pm 0.04$ & 0.1 & 0.15 & $48800 \pm 1200$ & 24,25 \\
\hline MT Ser & PN A66 41* & $\mathrm{sdO}, \mathrm{PN}$ & 0.113 & 0.6 & $0.2 \pm 0.1$ & $0.2 \pm 0.1$ & 50000 & 26,27 \\
\hline IN CMa & RE J0720-318 & WD & 1.26 & $0.57 \pm 0.02$ & $0.39 \pm 0.07$ & $0.375^{\%}$ & $53000 \pm 1100$ & 22 \\
\hline NN Ser & PG $1550+131$ & WD & 0.130 & $0.57 \pm 0.04$ & $0.12 \pm 0.03$ & $0.166^{\%}$ & $55000 \pm 8000$ & 28 \\
\hline TW Crv & EC 11575-1845 & $\mathrm{sdO}$ & 0.328 & & & & $95000 \pm 40000$ & 29 \\
\hline RE 1016-053 & & WD & 0.789 & $0.6 \pm 0.02$ & $0.15 \pm 0.02$ & $0.195^{\%}$ & $\begin{array}{l}55000 \pm 1000 \\
56400 \pm 1200\end{array}$ & $\begin{array}{l}22 \\
23\end{array}$ \\
\hline UU Sge & PN A66 63* & sdO,PN & 0.465 & $0.63 \pm 0.06$ & $0.29 \pm 0.03$ & $0.53 \pm 0.02$ & $\begin{array}{l}87000 \pm 13000 \\
57000 \pm 8000\end{array}$ & $\begin{array}{l}30,31,32 \\
32\end{array}$ \\
\hline V477 Lyr & PN A66 46* & sdOB,PN & 0.472 & $0.51 \pm 0.07$ & $0.15 \pm 0.02$ & $0.46 \pm 0.03$ & 60000 & 33 \\
\hline PN A66 65 & Abell 65 & sd?,PN & 1.00 & & & & 80000 & 34,35 \\
\hline KV Vel & LS 2018 & $\mathrm{sdO}, \mathrm{PN}$ & 0.357 & $0.63 \pm 0.03$ & $0.25 \pm 0.06$ & $0.402 \pm 0.005$ & $\begin{array}{l}77000 \pm 3000 \\
90000\end{array}$ & $\begin{array}{l}36 \\
37\end{array}$ \\
\hline HS $1136+6646$ & & WD & 1 & & & & 100000 & 38 \\
\hline BE UMa & PG $1155+492$ & sdO,PN & 2.291 & $0.7 \pm 0.07$ & $0.36 \pm 0.07$ & $0.72 \pm 0.05$ & $105000 \pm 5000$ & 39,40 \\
\hline
\end{tabular}

Other frequently used designations: * Abell 41, $46 \& 63$ (to be used with care as the object numbers in Abell 1955 and Abell 1966 are not consistent); ${ }^{\dagger} \mathrm{PG} 1224+309$.

! The radius of the secondary is estimated using the mass-radius relation shown by Kudritzki et al. (1982) (their Fig. 8).

\% Radii of the secondary calculated by using the mass-radius relation given by Politano (1996).

References: (1) Bruch (1999), (2) Bragaglia et al. (1995), (3) Bleach et al. (2000), (4) Kawka et al. (2002), (5) Marsh \& Duck (1996), (6) Maxted et al. (1998), (7) Saffer et al. (1993), (8) Kepler \& Nelan (1993), (9) Kawka et al. (2000), (10) Koester et al. (1979), (11) Stauffer (1987), (12) Lanning \& Pesch (1981), (13) Guinan \& Sion (1984), (14) Schmidt et al. (1995), (15) Wood \& Saffer (1999), (16) Drechsel et al. (2001), (17) Orosz et al. (1999), (18) Maxted et al. (2002), (19) O'Brien et al. (2001), (20) Kilkenny et al. (1998), (21) Rauch (2000), (22) Vennes et al. (1999), (23) Bergeron et al. (1994), (24) Fulbright et al. (1993), (25) Green et al. (1978), (26) Green et al. (1984), (27) Grauer \& Bond (1983), (28) Catalan et al. (1994), (29) Chen et al. (1995), (30) Miller et al. (1976), (31) Pollacco \& Bell (1993), (32) Bell et al. (1994), (33) Pollacco \& Bell (1994), (34) Bond \& Livio (1990), (35) Walsh \& Walton (1996), (36) Hilditch et al. (1996), (37) Herrero et al. (1990), (38) Sing et al. (2001), (39) Wood et al. (1995), (40) Ferguson et al. (1999).

systems are extremely close to the semi-detached state. Therefore Kawka et al. (2002) suggested that at least EC 13471-1258 might also be a hibernating nova instead of a PCEB. We additionally note that the radius obtained for the secondary in EC 13471-1258 is essentially lower than predicted by the main-sequence mass radius relation which appears implausible and indicates rather large uncertainties in the values for $M_{\mathrm{sec}}$ and/or $R_{\mathrm{sec}}$. Finally, the classification of the secondaries in PG 1017-086 and AA Dor is not unambiguous. It has been noted that in both systems the secondary may also be 

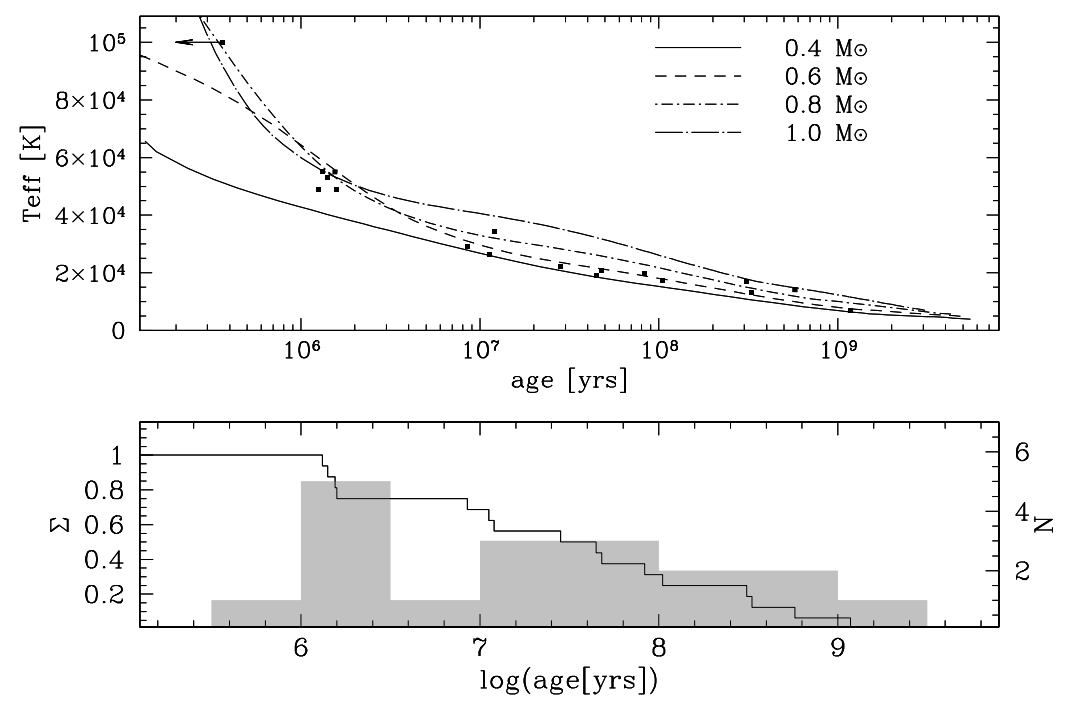

Fig. 4. Cooling tracks for non-accreting white dwarfs after Wood (1995) for different masses of the white dwarf. The position of the currently known PCEBs (Table 1) are obtained by interpolating between the cooling tracks for different masses of the primary. The bottom panel shows the age of the PCEB population in a histogram with logarithmic bins (shaded, N) and the relative cumulative distribution (solid line, $\Sigma$ ).

a brown dwarf rather than a low mass M-dwarf (Maxted et al. 2002; Rauch 2000).

\section{PCEBs: The past}

\subsection{The white dwarf cooling age}

The cooling of single white dwarfs has been modelled to a very high degree of precision allowing to derive accurate age estimates for field white dwarfs from their (observationally determined) effective temperatures and masses. Some uncertainties in the theoretical models remain at the low-temperature end of the white dwarf luminosity function, and are relevant primarily for the cosmo-chronological interpretation of the coldest (halo) white dwarfs (see the reviews by Fontaine et al. 2001; Koester 2002).

We determined the cooling ages for the PCEBs in our sample which contain a white dwarf primary by interpolating Wood's (1995) CO core evolution tracks for the effective temperatures and masses listed in Table $1^{3}$. The cooling age estimates are given in Table 2, and are shown in Fig. 4 along with Wood's cooling tracks. The bottom panel of Fig. 4 displays the age distribution of our PCEB sample. It is evident that the currently known population of PCEBs is strongly dominated by systems younger than $5 \times 10^{8} \mathrm{yrs}$, with the exception of RR Cae, which has a cooling age of $t_{\text {cool }} \sim 10^{9}$ yrs. The mean cooling age of the 18 systems in the sample containing a white dwarf primary is $\bar{t}_{\text {cool }} \sim 1.4 \times 10^{8}$ yrs. Considering that the sdOB, sdO, and PN systems in our sample are white dwarf progenitors, the mean age of the entire PCEB sample is even lower. The accuracy of our cooling age estimates depends (a) on the uncertainties in the white dwarf masses and (b) on the core

\footnotetext{
${ }^{3}$ Note that the same method can not be applied in CVs, as accretion (re)heats the white dwarf. As a result, white dwarfs in CVs are typically hotter than field white dwarfs of comparable age and mass (Gänsicke 2000; Townsley \& Bildsten 2002).
}

composition of the white dwarfs. We discuss both issues in more detail below.

(a) The white dwarf masses in PCEBs can be measured to a rather high level of precision, as several independent methods are available in these systems (fitting model spectra to the Balmer lines, dynamical measurements from the radial velocity variations of the white dwarf and its companion, measurement of the gravitational redshift of photospheric lines from the white dwarf). The errors on the white dwarf masses extracted from the literature (Table 1) are completely negligible in the context of our cooling age estimates. Even if we assume that the published errors are underestimated, and that the true errors in the white dwarf masses are of the order $\pm 0.1-0.2 M_{\odot}$, the corresponding uncertainties in the derived cooling ages are of the order $\sim 2$, which is still irrelevant for the comparison to the binary evolution time scales.

(b) The evolution of a white dwarf progenitor in a binary system may affect the core composition of the white dwarf. If the first Roche lobe overflow phase occurs prior to helium ignition in the primary star (de Kool \& Ritter 1993; Iben \& Tutukov 1993) the resulting white dwarf will be of low mass and have a helium core. Consequently, evolution models for $\mathrm{CVs}$ predict that systems with a low mass primary $\left(M_{1}<0.5 M_{\odot}\right)$ should contain a helium core white dwarf (de Kool \& Ritter 1993; de Kool 1992; Politano 1996; Howell et al. 2001). Careful observations of seven previously considered low mass single white dwarfs revealed that five of them are indeed close binary systems (Marsh et al. 1995).

Comparing the cooling models for CO white dwarfs (Wood 1995) and He white dwarfs (Althaus \& Benvenuto 1997; Driebe et al. 1998, 1999) shows, however, that the evolution of $\mathrm{CO}$ and He white dwarfs differs noticeably only for very low-mass stars, where residual hydrogen shell burning dominates over the gravothermal energy release. Inspecting Figs. 2 and 3 of Driebe et al. (1998) shows that only for a single system from our sample (Table 1) the cooling age derived from 
Wood's (1995) CO tracks may be significantly wrong: if the white dwarf in RR Cae $\left(0.467 M_{\odot}, 7000 \mathrm{~K}\right)$ contains a He core, then its cooling age is $\sim 4-5 \times 10^{9} \mathrm{yrs}$, compared to $1.2 \times 10^{9} \mathrm{yrs}$ for a CO core. We conclude that the effect of different core compositions is negligible in the context of the present paper, but has to be taken into account in future analyses if additional PCEBs containing low mass $\left(M_{1} \lesssim 0.4 M_{\odot}\right)$ and cool $\left(T_{\text {eff }} \lesssim 20000 \mathrm{~K}\right)$ white dwarfs are found.

A final note concerns the possible effect of re-accretion of CE material, which may alter the composition of the white dwarf envelope in a PCEB with respect to that of a single white dwarf. Differences in envelope/atmosphere composition will affect the cooling of the white dwarf only at the lowtemperature end of the luminosity function. While this effect is important for the cosmochronological interpretation of the coldest and oldest halo WDs (e.g. Koester 2002), it is irrelevant for our PCEB sample.

\subsection{The orbital period at the end of the common envelope phase}

During the detached post CE-phase the masses of the companions remain essentially constant, i.e. $\dot{M}_{1}=\dot{M}_{\text {sec }}=0$. With Kepler's third law this leads to the well known relation

$\frac{\dot{J}}{J}=\frac{\dot{P}}{3 P}$

Based on the the cooling age of a given PCEB derived from cooling tracks (Fig. 4, Table 2) we can approximate the orbital period at the end of the CE phase by integrating Eq. (7). We have done this calculation for both the standard scenario (Eq. (5)), and for the empirical AML prescription by Sills et al. (2000) (Eq. (6)). In the standard scenario magnetic braking is assumed to be much stronger than gravitational radiation for $M_{\text {sec }}>0.3 M_{\odot}$ and, hence, we can simply integrate Eq. (7) using either $\dot{J}=\dot{J}_{\mathrm{GR}}$ or $\dot{J}=\dot{J}_{\mathrm{VZ}}$ depending on the mass of the secondary. For gravitational radiation only we get:

$$
\begin{aligned}
t_{\mathrm{cool}}=\frac{5 c^{5}}{256 G^{5 / 3}(2 \pi)^{8 / 3}} \frac{\left(M_{1}+M_{\mathrm{sec}}\right)^{1 / 3}}{M_{1} M_{\mathrm{sec}}} \\
\times\left(P_{\mathrm{CE}}^{8 / 3}-P_{\mathrm{orb}}{ }^{8 / 3}\right),
\end{aligned}
$$

(see also Ritter 1986). For systems with $M_{\mathrm{sec}}>0.3 M_{\odot}$ we assume $\dot{J}=\dot{J}_{\mathrm{VZ}}$ and derive:

$$
\begin{aligned}
t_{\text {cool }}=\frac{2.63 \times 10^{29} G^{2 / 3} M_{1}}{(2 \pi)^{10 / 3}\left(M_{1}+M_{\mathrm{sec}}\right)^{1 / 3}} R_{\odot}^{-4}\left(\frac{R_{\odot}}{R_{\mathrm{sec}}}\right)^{\gamma} \\
\times\left(P_{\mathrm{CE}}^{10 / 3}-P_{\mathrm{orb}}{ }^{10 / 3}\right) .
\end{aligned}
$$

Considering the empirical angular momentum loss prescription of Sills et al. (2000) the situation is somewhat more complex as the two angular momentum loss agencies can be of the same order of magnitude (see Fig. 1) and we can not neglect gravitational radiation. Nevertheless, there exists also an analytical solution for the integral of Eq. (7) using $\dot{J}=\dot{J}_{\mathrm{RMB}}$ :

$$
\begin{aligned}
t_{\mathrm{cool}}=b\left[\frac{3\left(1+a P^{4 / 3}\right)}{4 a\left(P^{-5 / 3}+a P^{-1 / 3}\right) P^{1 / 3}}-\right. \\
\left.\frac{3\left(1+a P^{4 / 3}\right) \ln \left(1+a P^{4 / 3}\right)}{4 a^{2}\left(P^{-5 / 3}+a P^{-1 / 3}\right) P^{5 / 3}}\right]_{P_{\mathrm{CE}}}^{P_{\mathrm{orb}}}
\end{aligned}
$$

with

$$
\begin{aligned}
& b=\left(\frac{96 G^{5 / 3} M_{1} M_{\mathrm{sec}}(2 \pi)^{8 / 3}}{5 c^{5}\left(M_{1}+M_{\mathrm{sec}}\right)^{1 / 3}}\right)^{-1} \\
& a=\left(\frac{M_{\odot} R_{\mathrm{sec}}}{M_{\mathrm{sec}} R_{\odot}}\right)^{0.5} \frac{K_{w} \omega_{\mathrm{crit}^{2}}\left(M_{1}+M_{\mathrm{sec}}\right)^{2 / 3} 5 c^{5}}{32(2 \pi)^{4 / 3} G^{7 / 3} M_{1}^{2} M_{\mathrm{sec}}^{2}}
\end{aligned}
$$

Having derived the cooling age of the PCEBs we solve Eqs. (8), (9), and (10) to obtain the orbital period immediately after the $\mathrm{CE}$ phase $\left(P_{\mathrm{CE}}\right)$. Again, the results are given in Table 2. Not surprisingly, $P_{\mathrm{CE}}$ is very close to the present orbital period of the systems as we found most of the PCEBs to be quite young. For systems with $t_{\text {cool }} \lesssim 10^{7}$ yrs we get essentially $P_{\text {orb }} \simeq P_{\mathrm{CE}}$. Clearly, the assumed AML prescription makes a significant difference only for rather old PCEBs (i.e. RR Cae, in our sample) or for systems not too young and with $M_{\mathrm{sec}}>$ $0.3 M_{\odot}$ (EG UMa, V471 Tau, EC 13471-1258, BPM 71214). In these cases we give in Table 2 both results where the second line corresponds to $\dot{J}=\dot{J}_{\mathrm{RMB}}$. Comparing the obtained distribution of orbital periods after the CE-phase $\left(P_{\mathrm{CE}}\right)$ with theoretical predictions (Fig. 9 in de Kool 1992), we find reasonable agreement: for our systems we get $0.4>\log \left(P_{\mathrm{CE}}\right)>-1.1$ with $\sim 60 \%$ in the range of $-0.1>\log \left(P_{\mathrm{CE}}\right)>-0.6$.

\subsection{The total age of the PCEBs}

The cooling age of the primary represents just the age of the systems after the end of the CE. To approximate the total age of the binary, i.e. the time since it appeared on the main sequence we use simple analytical fits to the stellar evolution, Roche geometry, and Kepler's third law to obtain the nature of the initial main sequence binary. Combining Eqs. (35), (36), and (43)-(49) of Politano (1996) with the standard prescription of the binary shrinkage during the CE-phase (Eq. (8) in de Kool 1992) gives the mass of the initial primary and, hence, the nuclear evolution time. This is, of course, just a rough approximation because an uncertain fraction $\alpha_{\mathrm{CE}}$ of the gravitational binding energy that is released when the secondary and the primary spiral together goes into the ejection of the envelope. However, we assume $\alpha_{\mathrm{CE}}=1$ and $\lambda=0.5$ (see de Kool 1992) which gives results in reasonable agreement with the initialto-final mass relation for CO-white dwarfs (Weidemann 2000). The estimates obtained for the masses of the progenitors of the primaries $\left(M_{1, \mathrm{pCE}}\right)$ and the resulting evolution time since the binary appeared on the main sequence $\left(t_{\text {evol }}\right)$ are also given in Table 2. For PCEBs containing a low mass white dwarf $t_{\text {evol }}$ can be of the order of $10 \%$ of the Hubble time (see Table 2) and therefore is not generally negligible. 


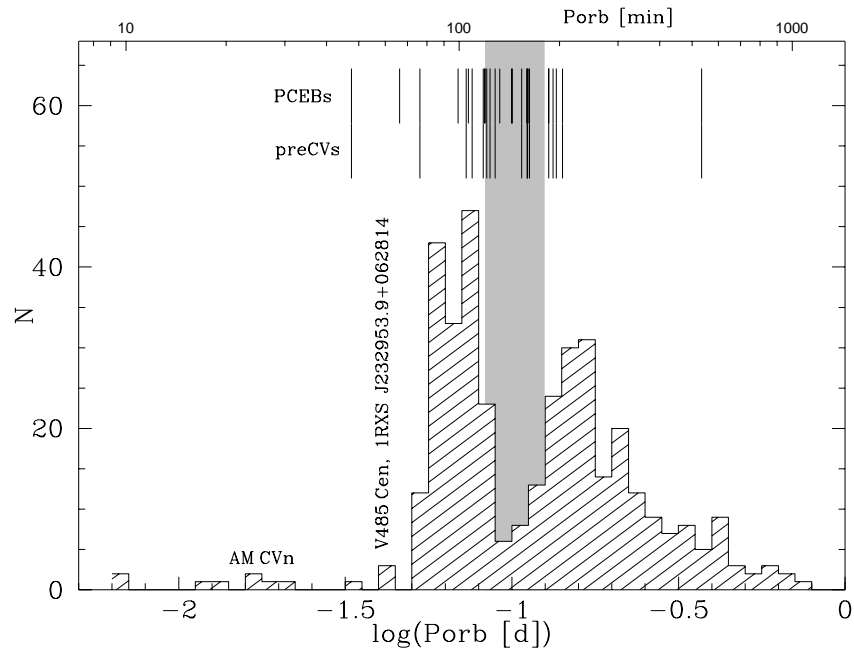

Fig. 5. The orbital period distribution of the currently known CV population (from Kube et al. 2002). Grey-shaded is the orbital period gap. The vertical lines on the top indicate the periods at which the PCEBs from Table 1 will enter a semi-detached configuration and start mass transfer. Those PCEBs that will evolve into CVs within less than a Hubble time (Table 2) are called pre-CVs (see Sect. 1). The two PCEBs with $P_{\text {sd }}$ below the minimum orbital period of CVs at $\sim 80 \mathrm{~min}$ are the two systems in our sample which possibly have a brown dwarf secondary AA Dor and PG 1017-086 (see Sect. 4).

\section{PCEBs: The future}

\subsection{How long before the onset of mass transfer?}

In the last section we showed that the vast majority of the known PCEBs are young objects. Here, we calculate the orbital periods that these binaries will have when they turn into CVs as well as the time scale for this evolution.

Knowing $M_{1}$ and $M_{\text {sec }}$ of a (detached) PCEB (Table 1) the orbital period of the corresponding semi-detached configuration, $P_{\text {sd }}$ follows from Roche geometry and Kepler's third law (Ritter 1986):

$$
P_{\text {sd }}=9 \pi\left(\frac{R_{\text {sec }}{ }^{3}}{G M_{\mathrm{sec}}\left(1+M_{1} / M_{\mathrm{sec}}\right)\left(R_{\mathrm{L}} / a\right)^{3}}\right)^{0.5} .
$$

Here $R_{\mathrm{L}}$ denotes the volume radius of the Roche-lobe of the secondary and $a$ the binary separation. We use the formula of Eggleton (1983) to relate these quantities to the mass ratio of the system. The resulting $P_{\text {sd }}$ for the PCEBs in our sample are listed in Table 2 and are compared to the orbital period distribution of the currently known CV population in Fig. 5. It is apparent that only a small number of systems $(\sim 22 \%)$ of the currently known PCEB population will evolve into CVs above the period gap $\left(P_{\mathrm{sd}}>3 \mathrm{~h}\right)$, whereas $\sim 30 \%$ will evolve into contact at $P_{\mathrm{sd}}<2 \mathrm{~h}$. A somewhat surprising result is that almost half $(\sim 48 \%)$ of the currently known PCEBs will start their CV life in the period gap $\left(2 \mathrm{~h}<P_{\text {sd }}<3 \mathrm{~h}\right)$. The small number of progenitors of long-period CVs will be discussed in Sect. 9.

Knowing $P_{\text {sd }}$ we now calculate the time it will take a PCEB to become a $\mathrm{CV}\left(t_{\mathrm{sd}}\right)$, again by integrating Eq. (7) assuming either AML according to the "standard scenario" $\left(\dot{J}=\dot{J}_{\mathrm{CMB}}\right)$ or Sill's et al. (2000) empiric AML prescription $\left(\dot{J}=\dot{J}_{\mathrm{RMB}}\right)$. The solution for $t_{\mathrm{sd}}$ can be derived by replacing $t_{\mathrm{cool}}, P_{\mathrm{orb}}, P_{\mathrm{CE}}$ with $t_{\mathrm{sd}}, P_{\mathrm{sd}}, P_{\text {orb }}$ in Eqs. (8-9). It is worth to note that although the PCEBs evolve on a time scale comparable or even longer than $10^{10} \mathrm{yrs}$, the expansion of the secondary due to its nuclear evolution is negligible for all the PCEBs in our sample and, hence, not taken into account in our calculations. Inspecting the resulting $t_{\mathrm{sd}}$ (Table 2), it becomes evident that most of the PCEBs have completed only a small fraction $f_{\mathrm{PCEB}}$ of their predicted PCEB-lifetime. On average the total evolution time for the PCEBs is $\sim 3.4 \times 10^{10} \mathrm{yrs}$ independent on the assumed AML prescription ${ }^{4}$.

Assuming $\dot{j}=\dot{J}_{\mathrm{CMB}}$, four systems (i.e. EGUMa, EC 13471-1258, BPM 71214, V471 Tau) have $f_{\mathrm{PCEB}}>0.1$. EC 13471-1258 is expected to be very close to the onset of mass transfer, $f_{\mathrm{PCEB}}>0.995$, and one may speculate that this system is an old nova rather than a PCEB (Kawka et al. 2002, see also Sect. 4).

As expected, $t_{\mathrm{sd}}$ of an individual system depends strongly on the assumed AML prescription. Following Andronov et al. (2003) we obtain shorter evolution times (by a factor of 1.5-4) compared to the standard scenario for systems with $M_{\mathrm{sec}}<$ $0.3 M_{\odot}$, whereas for systems with $M_{\mathrm{sec}} \geq 0.3 M_{\odot} t_{\mathrm{sd}}$ is significantly longer (by a factor of 3-30 depending on the present orbital period of the system). The differences in $t_{\mathrm{sd}}$, depending on the use of $\dot{J}_{\mathrm{CMB}}$ or $\dot{J}_{\mathrm{RMB}}$ are illustrated for the systems HR Cam and EG UMa in Fig. 6.

Figure 7 shows that most of the PCEB white dwarfs will be very cool when the secondary is expected to fill its Roche-lobe and mass transfer starts. Only for $\dot{J}_{\mathrm{CMB}}$ we get one system, (i.e. V471 Tau, the filled square in the upper left corner of Fig. 7) in which the mass transfer starts while the primary still can be considered a "hot" white dwarf.

\subsection{Representative progenitors of the current CV population}

Analysing PCEBs is not only interesting in itself, it is also important in the context of $\mathrm{CV}$ formation and evolution (see Sects. 1 and 2). Discussing the properties of our sample of PCEBs in the framework of the present-day CV population requires to select those systems which are representative for the former progenitors of the current $\mathrm{CV}$ population. Only PCEBs which will evolve into CVs in less than the Hubble time (assuming $\tau_{0}=1.3 \times 10^{10} \mathrm{yrs}^{5}$ ) satisfy this condition. To distinguish these systems from the PCEBs which are different from the former progenitors of the present $\mathrm{CVs}$ we refer to them as pre-CVs (see also Sect. 1).

Applying this selection criterion, we find 14 (16) pre$\mathrm{CV}$ candidates in our sample (Table 2) when assuming

\footnotetext{
4 Notice, $\dot{J}_{\mathrm{RMB}}$ is smaller than $\dot{J}_{\mathrm{CMB}}$ only above the gap otherwise $\dot{J}_{\mathrm{RMB}}$ is more efficient.

5 Recently Ferreras et al. (2001) set new constraints on the age of the universe combining the results obtained by cosmochromology, stellar population synthesis, and mapping of the peaks in the microwave background. They derived an age of $13.2_{-0.8}^{+1.2}$ Gyrs. Note also that Cayrel et al. (2001) obtained a stellar age of $12.5 \pm 3$ Gyrs from uranium decay. Throughout this work we therefore assume that star formation in the Galaxy began 13 Gyrs ago.
} 
Table 2. The properties of the PCEBs calculated in Sects. 5-7. If there is no reference given in the table, the distance is obtained from the references listed in Table 1. The time until the mass transfer is expected to start $\left(t_{\mathrm{sd}}\right)$ is calculated using Eqs. (8), (9) (CMB) and Eq. (10) (RMB). The total time until the system becomes semi-detached, $t_{\text {tot }}$ is given by the sum of the present cooling age, $t_{\text {cool }}$, the estimated MS lifetime of the primary, $t_{\mathrm{evol}}$, and $t_{\mathrm{sd}}$. PCEBs with $t_{\mathrm{tot}}$ less than a Hubble time are marked in bold. For the systems RR Cae, EG UMa, EC 13471-1258, BPM 71214 and V471 Tau we find the properties of the progenitor of the PCEB (orbital period $P_{\mathrm{CE}}$, primary mass $M_{1, \mathrm{pCE}}$ and, $t_{\mathrm{evol}}$ ) depending on the assumed AML (see text). In these cases, the second line gives the results assuming reduced magnetic braking (RMB) whereas the first line corresponds to the classical magnetic braking assumption (CMB). $f_{\mathrm{PCEB}}$ gives the mean fractional PCEB life time that the system has already passed through. The orbital period at which the PCEB starts mass transfer $P_{\mathrm{sd}}$ is independent on the assumed angular momentum loss.

\begin{tabular}{|c|c|c|c|c|c|c|c|c|c|c|c|c|c|}
\hline \multirow[b]{2}{*}{ Object } & \multirow[b]{2}{*}{$d[\mathrm{pc}]$} & \multirow[b]{2}{*}{ Ref. } & \multirow[b]{2}{*}{$\log \left(t_{\text {cool }}\right)$} & \multirow[b]{2}{*}{$P_{\mathrm{CE}}[\mathrm{d}]$} & \multirow[b]{2}{*}{$\log \left(t_{\text {evol }}\right)$} & \multirow[b]{2}{*}{$M_{1, \mathrm{pCE}}$} & \multirow[b]{2}{*}{$\log \left(t_{\mathrm{sd}}\right)$} & \multirow{2}{*}{$\begin{array}{r}\mathrm{CMB} \\
f_{\mathrm{PCEB}} \\
\end{array}$} & \multirow[b]{2}{*}{$\log \left(t_{\mathrm{tot}}\right)$} & \multicolumn{3}{|c|}{ RMB } & \multirow[b]{2}{*}{$P_{\mathrm{sd}}[\mathrm{d}]$} \\
\hline & & & & & & & & & & $\log \left(t_{\mathrm{sd}}\right)$ & $f_{\mathrm{PCEB}}$ & $\log \left(t_{\mathrm{tot}}\right)$ & \\
\hline \multirow[t]{2}{*}{ RR Cae } & 11 & 1 & 9.07 & 0.307 & 9.48 & 1.41 & 10.54 & 0.033 & 10.58 & 10.26 & 0.061 & 10.35 & 0.100 \\
\hline & & & & 0.312 & 9.49 & 1.41 & & & & & & & \\
\hline \multirow[t]{2}{*}{ EG UMa } & $32 \pm 5$ & 2 & 8.52 & 0.764 & 8.78 & 2.58 & 8.76 & 0.365 & 9.18 & 10.22 & 0.020 & 10.24 & 0.142 \\
\hline & & & & 0.675 & 8.76 & 2.63 & & & & & & & \\
\hline \multirow[t]{2}{*}{ EC 13471-1258 } & 55 & & 8.57 & 0.583 & 8.34 & 3.98 & 6.23 & 0.995 & 8.77 & 8.16 & 0.720 & 8.82 & 0.129 \\
\hline & & & & 0.197 & 8.18 & 4.71 & & & & & & & \\
\hline \multirow[t]{2}{*}{ ВРM 71214} & 68 & & 8.33 & 0.466 & 8.38 & 3.79 & 7.00 & 0.955 & 8.67 & 8.97 & 0.186 & 9.12 & 0.136 \\
\hline & & & & 0.212 & 8.25 & 4.37 & & & & & & & \\
\hline HR Cam & $72 \pm 11$ & 2,3 & 7.65 & 0.104 & 9.43 & 1.48 & 9.25 & 0.025 & 9.66 & 9.16 & 0.030 & 9.62 & 0.053 \\
\hline UZSex & $35 \pm 5$ & 2 & 7.92 & 0.599 & 8.86 & 2.39 & 10.90 & 0.001 & 10.90 & 10.45 & 0.003 & 10.46 & 0.100 \\
\hline ВРМ 6502 & $25 \pm 4$ & 2 & 7.68 & 0.338 & 8.66 & 2.88 & 10.44 & 0.002 & 10.45 & 10.08 & 0.004 & 10.10 & 0.086 \\
\hline \multirow[t]{2}{*}{$\mathrm{HZ} 9$} & $40 \pm 6$ & 2 & 8.02 & 0.567 & 8.66 & 2.88 & 10.82 & 0.002 & 10.82 & 10.26 & 0.006 & 10.27 & 0.112 \\
\hline & 46 & 3 & & & & & & & & & & & \\
\hline MS Peg & 61 & & 7.45 & 0.175 & 9.00 & 2.12 & 9.42 & 0.011 & 9.56 & 9.18 & 0.018 & 9.40 & 0.111 \\
\hline CCCet & $89 \pm 13$ & 2 & 7.05 & 0.284 & 9.67 & 1.22 & 10.27 & 0.001 & 10.36 & 9.88 & 0.001 & 10.09 & 0.084 \\
\hline HW Vir & $171 \pm 19$ & & & 0.118 & 9.05 & 2.02 & 9.12 & & 9.38 & 8.99 & & 9.32 & 0.076 \\
\hline HS 0705+6700 & 1150 & 2 & & 0.096 & 9.01 & 2.11 & 8.61 & & 9.16 & 8.48 & & 9.12 & 0.082 \\
\hline LM Com & $\begin{array}{l}170 \pm 26 \\
290\end{array}$ & 2 & 7.05 & 0.259 & 9.14 & 1.87 & 9.91 & 0.001 & 9.98 & 9.55 & 0.003 & 9.69 & 0.111 \\
\hline PG 1017-086 & 990 & 2 & & 0.073 & 8.66 & 2.88 & 8.91 & & 9.10 & 8.89 & & 9.09 & 0.033 \\
\hline V471 Tau & $47 \pm 4$ & 3 & 6.93 & 0.584 & 8.11 & 5.08 & 7.54 & 0.197 & 8.23 & 8.89 & 0.011 & 8.91 & 0.371 \\
\hline & & & & 0.522 & 8.10 & 5.12 & & & & & & & \\
\hline NY Vir & $710 \pm 50$ & & & 0.101 & 8.66 & 2.88 & 8.51 & & 8.89 & 8.37 & & 8.84 & 0.089 \\
\hline & 560 & 2 & & & & & & & & & & & \\
\hline AA Dor & 396 & & & & & & 10.65 & & & 10.51 & & & 0.046 \\
\hline RE $2013+400$ & $95-125$ & & 7.08 & 0.706 & 8.64 & 2.95 & 11.22 & $<0.001$ & 11.22 & 10.65 & $<0.001$ & 10.65 & 0.092 \\
\hline GK Vir & $350 \pm 50$ & 2 & 6.20 & 0.344 & 8.66 & 2.89 & 10.65 & $<0.001$ & 10.65 & 10.37 & $<0.001$ & 10.38 & 0.069 \\
\hline MT Ser & 4300 & 4 & & 0.113 & 8.62 & 3.00 & 8.86 & & 9.06 & 8.76 & & 9.00 & 0.074 \\
\hline & 4510 & 5 & & & & & & & & & & & \\
\hline & 5400 & 6 & & & & & & & & & & & \\
\hline & 4600 & 7 & & & & & & & & & & & \\
\hline IN CMa & $158-208$ & & 6.10 & 1.260 & 8.64 & 2.96 & 9.71 & $<0.001$ & 9.75 & 10.68 & $<0.001$ & 10.68 & 0.133 \\
\hline & 186 & 8 & & & & & & & & & & & \\
\hline NN Ser & $356-417$ & 9 & 6.12 & 0.130 & 8.64 & 2.96 & 9.32 & 0.001 & 9.40 & 9.20 & 0.001 & 9.31 & 0.073 \\
\hline TW Crv & 557 & & & & & & & & & & & & \\
\hline RE 1016-053 & 75-109 & & 6.19 & 0.790 & 8.62 & 3.00 & 11.40 & $<0.001$ & 11.40 & 10.83 & $<0.001$ & 10.83 & 0.083 \\
\hline UU Sge & $2400 \pm 400$ & & & 0.465 & 8.60 & 3.05 & 10.50 & & 10.51 & 10.10 & & 10.11 & 0.113 \\
\hline & 3600 & 10 & & & & & & & & & & & \\
\hline & 2150 & 5 & & & & & & & & & & & \\
\hline V477 Lyr & $1700 \pm 600$ & & & 0.472 & 8.66 & 2.89 & 10.86 & & 10.86 & 10.40 & & 10.41 & 0.083 \\
\hline & 1850 & 5 & & & & & & & & & & & \\
\hline & 2190 & 8 & & & & & & & & & & & \\
\hline PN A66 65 & 1100 & 5 & & & & & & & & & & & \\
\hline & 1660 & 8 & & & & & & & & & & & \\
\hline KV Vel & $730-1000$ & 11 & & 0.357 & 8.60 & 3.05 & 10.25 & & 10.25 & 9.94 & & 9.95 & 0.107 \\
\hline HS $1136+6646$ & & & 5.55 & & & & & & & & & & \\
\hline BE UMa & 2000 & & & 2.291 & 8.85 & 2.42 & 10.70 & & 10.71 & 11.17 & & 11.71 & 0.129 \\
\hline
\end{tabular}

References: (1) Bruch \& Diaz (1998), (2) this work, Sect. 7, (3) Perryman et al. (1998), (4) Abell (1966), (5) Cahn \& Kaler (1971), (6) Maciel (1984), (7) Cahn et al. (1992), (8) Vennes \& Thorstensen (1996), (9) Wood \& Marsh (1991), (10) Walton et al. (1993), (11) Pottasch (1996). 

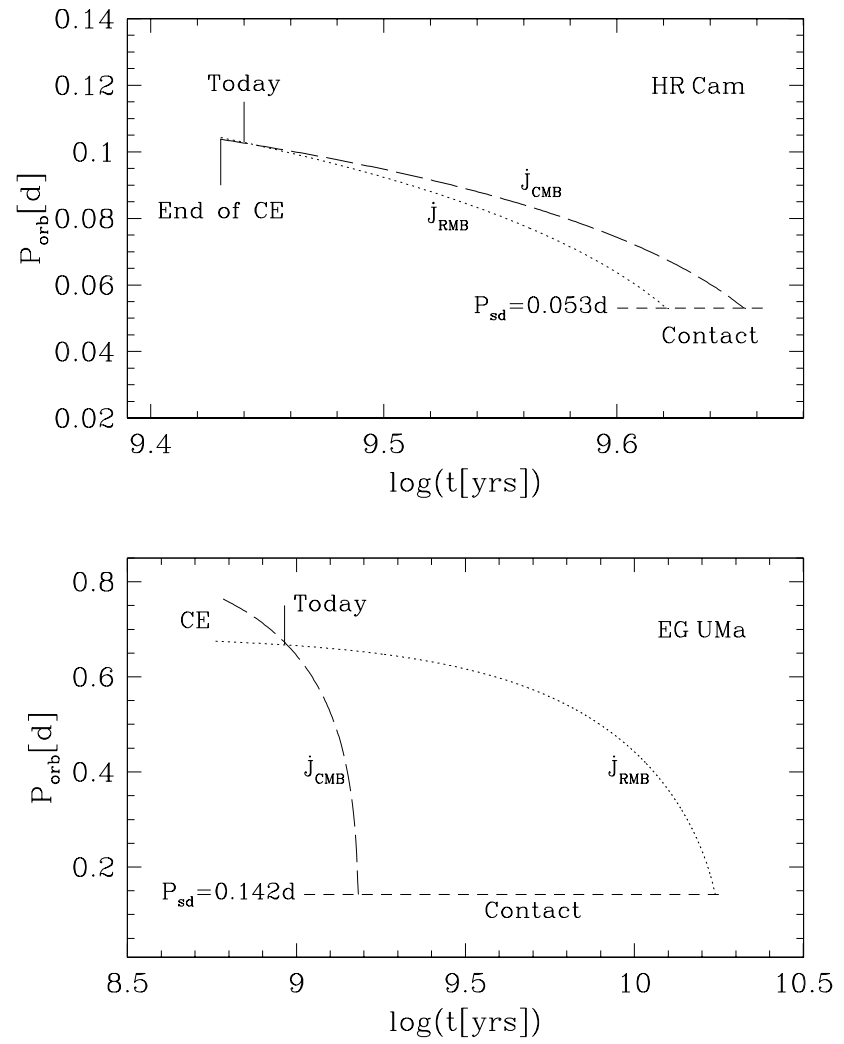

Fig. 6. The PCEB evolution of HR Cam and EG UMa assuming two different prescriptions for the AML, $\dot{J}_{\mathrm{CMB}}$ and $\dot{J}_{\mathrm{RMB}}$. As the mass of the secondary in HR Cam is significantly below $0.3 M_{\odot}$ the system evolves slower into contact if we assume AML according to the standard scenario. Conversely, EG UMa turns into a CV much faster in the standard scenario because of the more efficient magnetic braking for $M_{\mathrm{sec}}>0.3 M_{\odot}$.

$\dot{J}_{\mathrm{CMB}}\left(\dot{J}_{\mathrm{RMB}}\right)$. Although $t_{\mathrm{sd}}$ depends strongly on the assumed AML for some individual systems (e.g. EG UMa, Fig. 6; see also Fig. 8), the number of pre-CVs among the PCEB sample is nearly independent on it (see Table 2, Fig. 8). From the sample of pre-CVs 5 (3) will initiate mass transfer at orbital periods $>3 \mathrm{~h}$ whereas the other 9 (13) systems will start their CV life in or below the orbital period gap. This finding is in good agreement with the prediction of King et al. (1994) that $\gtrsim 67 \%$ of CVs will start mass transfer near or below the period gap. Possible observational selection effects will be discussed in Sect. 9.

A further test on whether the current pre-CV population is a representative sample of progenitors for the present $\mathrm{CV}$ population comes from comparing their space density to that of CVs. An estimate of the space density of PCEBs/pre-CVs requires obviously the knowledge of their distances.

\section{Distance estimates}

We estimate the distances to the systems in Table 1 for which no previous distance determination has been available in the literature using the following methods.

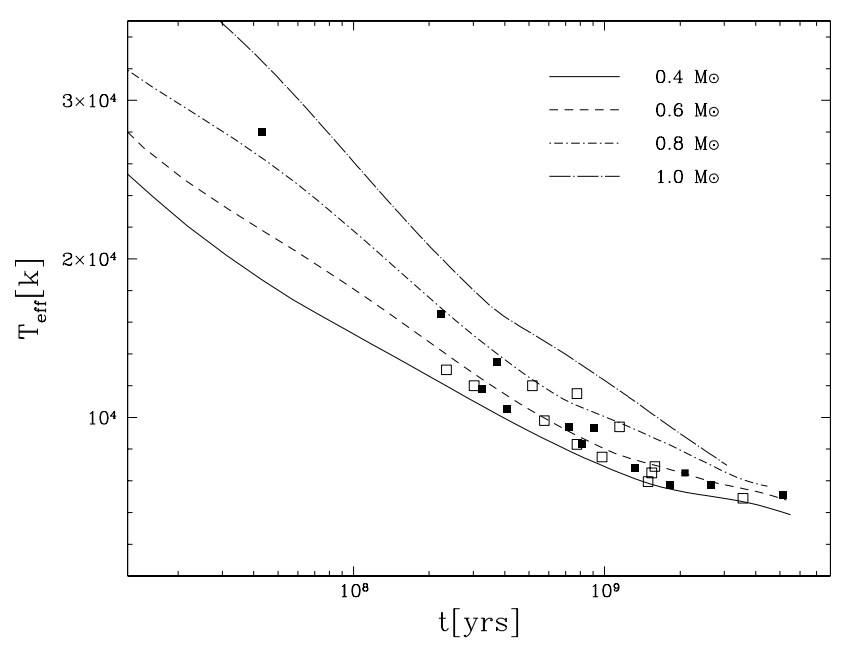

Fig. 7. The cooling tracks of Wood (1995) and the locations in the age/temperature plane where mass transfer will initiate for the PCEBs with a short PCEB lifetime $\left(t_{\text {cool }}+t_{\mathrm{sd}}<6 \times 10^{9} \mathrm{yrs}\right)$. The filled squares correspond to $\dot{J}_{\mathrm{CMB}}$ the open squares to $\dot{J}_{\mathrm{RMB}}$. Notice, most of the PCEBs will stay more than $10^{10} \mathrm{yrs}$ in the PCEB state and, hence, will have $T_{\text {eff }}<6000 \mathrm{~K}$ when becoming semi-detached.

\subsection{PCEBs with a white dwarf primary}

For all but one system without a published distance estimate ultraviolet (UV) spectroscopy is available in the public archives of the International Ultraviolet Explorer (IUE) and the Hubble Space Telescope. We fitted the UV data listed by Table 3 with pure-hydrogen white dwarf model spectra computed with the code described by Gänsicke et al. (1995), fixing $\log g=8.0$, i.e. $M_{1} \approx 0.6 M_{\odot}$. The free parameters of this fit were, hence, the white dwarf temperature $T_{\text {eff }}$ and the scaling factor $f / H=$ $4 \pi R_{1}{ }^{2} / d^{2}$, with $f$ the observed flux and $H$ the Eddington flux of the model spectra. The results from our fits are given in Table 3. Overall, the white dwarf temperatures that we derived here are in good agreement with the published values.

Fixing the surface gravity (= white dwarf mass) in the fit introduces a systematic uncertainty in the derived distances, as the white dwarf radius obviously depends on the assumed mass. However, it is important to notice that also the white dwarf temperature derived from the fit depends on the assumed mass, with higher (lower) temperatures resulting for higher (lower) $\log g$. This effect compensates to some extent the $R_{1}\left(M_{1}\right)$ dependence, and the error in the spectroscopic parallaxes is $\simeq \pm 15 \%$ for an assumed range $\log g=8.0 \pm 0.5$ (i.e. $0.35 M_{\odot} \lesssim M_{1} \lesssim 0.9 M_{\odot}$ ), which covers probably most of the analysed objects. The Hyades member HZ 9 can be used to estimate the robustness of our distance estimate and error analysis. From our model fit to unpublished HST STIS spectroscopy, we find a temperature which is slightly below the low end of the range quoted by Guinan \& Sion (1984), which was based on the analysis of $I U E$ spectroscopy. However, the much better $H S T$ data available now clearly shows the $1400 \AA$ quasimolecular $H_{2}+$ absorption which indicates a temperature $<20000 \mathrm{~K}$ (Fig. 9). We derive from the scaling factor of the model a distance of $d=40 \pm 6 \mathrm{pc}$, which is entirely consistent with the Hipparcos-measured parallax (Perryman et al. 1998). 


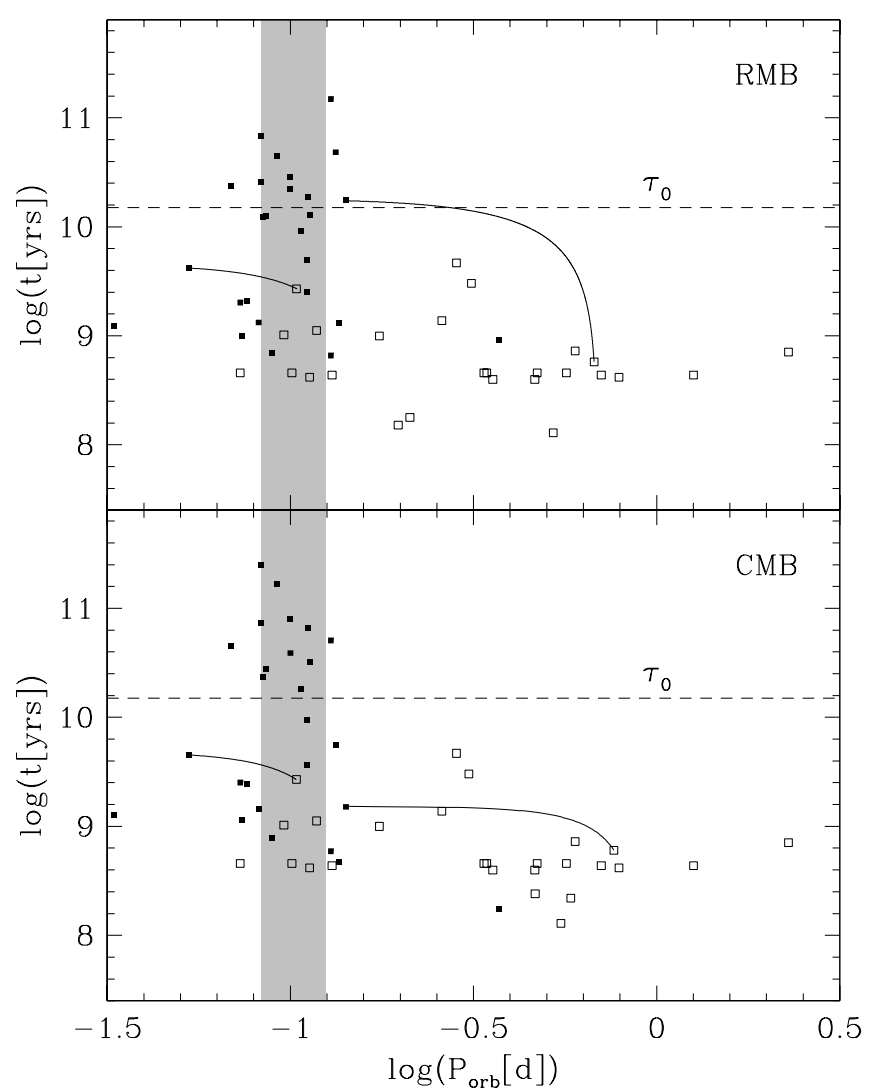

Fig. 8. Age of the binary versus orbital period for $\dot{J}_{\mathrm{CMB}}$ (bottom panel) and $\dot{J}_{\mathrm{RMB}}$ (top panel). The open squares mark the positions of the PCEBs immediately after the CE-phase $\left(P_{\mathrm{CE}}, t_{\mathrm{evol}}\right)$ whereas the filled squares indicate the positions when the mass transfer starts $\left(P_{\mathrm{sd}}\right.$, $\left.t_{\text {evol }}+t_{\text {cool }}+t_{\mathrm{sd}}\right)$. The solid lines show the orbital evolution of EG UMa and HR Cam. The dashed horizontal line indicates the age of the universe $\tau_{0} \sim 1.3 \times 10^{10} \mathrm{yrs}$ (see text).

For GK Vir, we had to fix the temperature to the value derived by Fulbright et al. (1993) as the temperature-sensitive Ly $\alpha$ absorption line is entirely blended with geocoronal emission in the available IUE spectrum.

A final note concerns the distance of LM Com. Orosz et al. (1999) quote a distance of 290-308 pc, which they base on a lengthy discussion of the white dwarf contribution to the observed $R$ band magnitude. However, as the contamination of the M-dwarf is lowest in the blue, we re-examined the distance of LMCom scaling a $T_{\text {eff }}=30000 \mathrm{~K}, \log g=8.0$ model to $F_{\lambda}(4700 \AA) \simeq 17 \times 10^{-16} \mathrm{erg} \mathrm{cm}^{-2} \mathrm{~s}^{-1} \AA^{-1}$ (measured from Orosz et al. 1999, Fig. 7), and find $d=170$ pc.

\subsection{PCEBs containing a $s d B$ primary}

Considering the rather similar properties of the sdB primaries in HW Vir, HS 0705+6700, PG 1017-086, and NY Vir $\left(M_{1}\right.$, $T_{\text {eff }}$, see Table 1), we computed (crude) distances estimates by scaling the distance of HW Vir $(d=171 \mathrm{pc}, V=10.6$; Wood \& Saffer 1999) to the observed $V$ magnitudes of the other sdB PCEBs (HS 0705+6700: $V=14.8$; PG 1017-086: $V=14.4$; NY Vir: $V=13.2$ ). This estimate ignores possible extinction as well as differences in $T_{\text {eff }}$ and $R_{1}$.

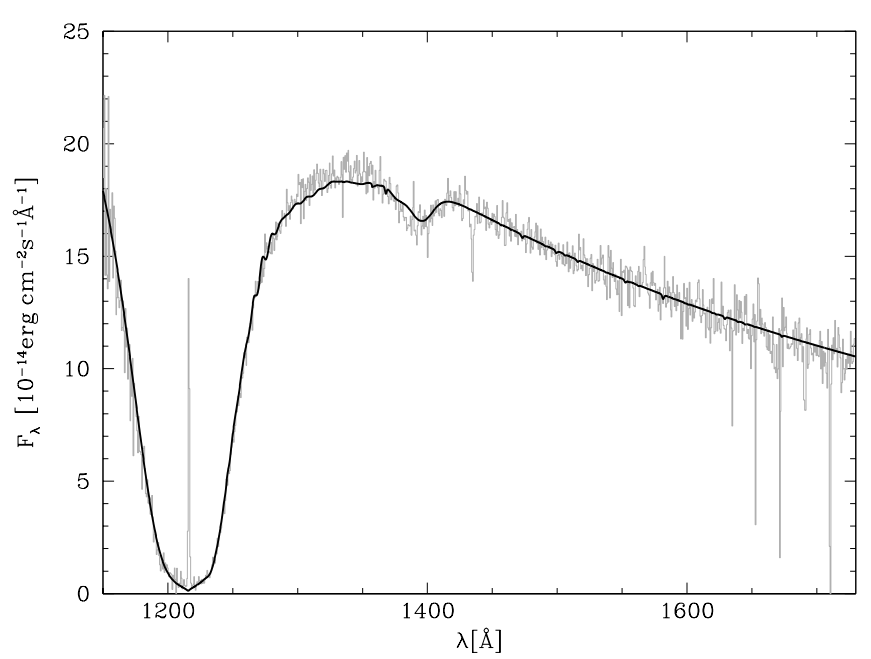

Fig. 9. The HST/STIS echelle spectrum of HZ9 along with the bestfit $T_{\text {eff }}=17400 \mathrm{~K}$ pure hydrogen white dwarf model, assuming $\log g=8.0$. The sharp "absorption dips" at the red end of the spectrum are due to gaps between the echelle orders.

Table 3. Distance estimates for PCEBs, see text.

\begin{tabular}{lrrrr}
\hline \hline Object & Instrument & Dataset & $T_{\text {eff }}[\mathrm{K}]$ & $d[\mathrm{pc}]$ \\
\hline EG UMa & HST/FOS & y16u0502t & 13400 & 32 \\
HR Cam & HST/STIS & o6gj02020 & 20800 & 72 \\
UZ Sex & IUE & swp27393 & 17200 & 35 \\
BPM 6502 & IUE & swp27351 & 21400 & 25 \\
HZ 9 & HST/STIS & o5dma6010 & 17400 & 40 \\
CC Cet & IUE & swp27392 & 25000 & 89 \\
GK Vir & IUE & swp07459 & $48800^{*}$ & 350 \\
\hline
\end{tabular}

*Temperature fixed to the value given by Fulbright et al. (1993).

\section{Space densities}

Inspection of Table 2 shows that 12 PCEBs from our sample are within $100 \mathrm{pc}$ (including RE 1016-053, $d=75-109 \mathrm{pc}$ ). This gives an "observed" space density of $\left(=2.9 \times 10^{-6} \mathrm{pc}^{-3}\right)$ which can be considered to be a conservative lower limit on the PCEB space density as the presently known PCEB sample is systematically dominated by young PCEBs (see Sect. 5.1, Table 2). Accounting for this bias, we estimate the true PCEB space density to be:

$\rho_{\mathrm{PCEB}} \simeq 2.9 \times 10^{-6} \frac{0.5}{\bar{f}_{\mathrm{PCEB}}}= \begin{cases}6.6 \times 10^{-6} \mathrm{pc}^{-3} & \left(\dot{J}_{\mathrm{CMB}}\right) \\ 2.9 \times 10^{-5} \mathrm{pc}^{-3} & \left(\dot{J}_{\mathrm{RMB}}\right)\end{cases}$

where $\bar{f}_{\text {PCEB }}$ is the mean fractional PCEB life time that the systems have already passed through. The substantial difference between the estimates of $\rho_{\text {PCEB }}$ for the two AML prescriptions results from the fact that the mean evolution time scale is significantly longer for $\dot{J}=\dot{J}_{\mathrm{RMB}}$ and, thus, the existence of more old and yet undetected PCEBs is predicted. However, even the higher value of $\rho_{\mathrm{PCEB}}$, assuming $\dot{J}_{\mathrm{RMB}}$, is still significantly below the theoretical predictions of de Kool \& Ritter (1993, their Fig. 4) indicating that our sample is probably not only biased towards young systems (see Sect. 9.3). 


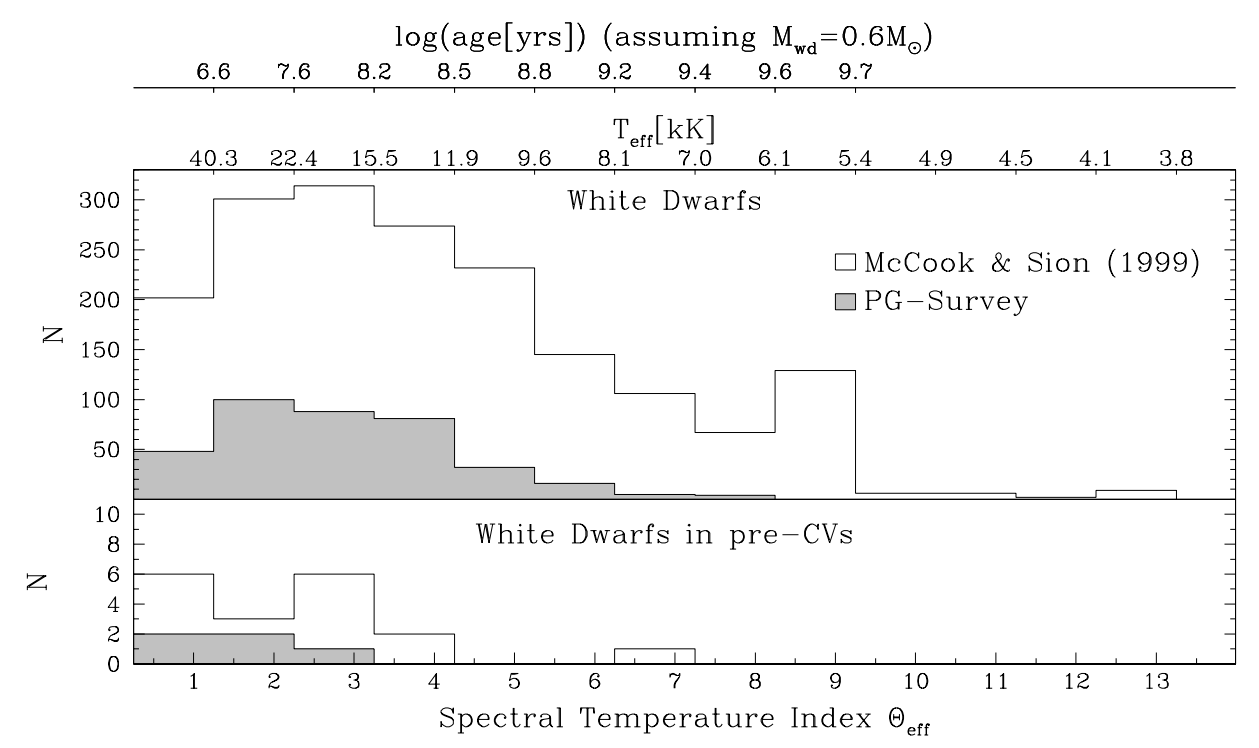

Fig. 10. The currently known populations of single white dwarfs (top) and PCEBs (bottom) as a function of their effective temperature. Here, we follow McCook \& Sion (1999) and plot the spectral temperature index, $\Theta_{\text {eff }}=50400 / T_{\text {eff }}$, which is a direct indicator of the age of the white dwarfs. The grey shaded histograms represent systems which have been found in the PG survey. Obviously, there exists a population of cool single white dwarfs whereas PCEBs white dwarfs are generally hot and young.

Requiring $t_{\text {tot }}<\tau_{0}$ for PCEBs which can be considered pre$\mathrm{CVs}$, we find $6\left(\dot{J}_{\mathrm{CMB}}\right)$ and $7\left(\dot{J}_{\mathrm{RMB}}\right)$ of these systems within $100 \mathrm{pc}$, resulting in lower limits on their space density $\rho_{\text {preCv }}>$ $1.4 \times 10^{-6} \mathrm{pc}^{-3}\left(\dot{J}_{\mathrm{CMB}}\right)$ and $\rho_{\mathrm{preCV}}>1.7 \times 10^{-6} \mathrm{pc}^{-3}\left(\dot{J}_{\mathrm{RMB}}\right)$. We estimate the actual pre-CV space density, again taking into account age and evolution time scale and find

$\rho_{\text {preCV }} \simeq \begin{cases}1.7 \times 10^{-6} \mathrm{pc}^{-3} & \left(\dot{J}_{\mathrm{CMB}}\right) \\ 6.0 \times 10^{-6} \mathrm{pc}^{-3} & \left(\dot{J}_{\mathrm{RMB}}\right)\end{cases}$

Our estimate of the current pre-CV space density allows an estimate for the present CV space density. Considering the average of the pre-CV lifetime ( $\left.\bar{t}_{\mathrm{preCV}}\right)$ of the systems we find within $100 \mathrm{pc}$ and following Politano (1996) in assuming that the $\mathrm{CV}$ birthrate has been constant since $t_{\mathrm{gal}}=10^{10} \mathrm{yrs}$ we get

$\rho_{\mathrm{CV}} \sim t_{\mathrm{gal}} \frac{\rho_{\mathrm{preCV}}}{\bar{t}_{\mathrm{preCV}}} \sim 1 \times 10^{-5} \mathrm{pc}^{-3}$

for both angular momentum loss prescriptions.

Considering the assumptions involved and the small number statistics of the known pre-CV sample, this result has to be regarded as a rather rough estimate. Nevertheless, our estimate for $\rho_{\mathrm{CV}}$ is in agreement with the high end of current observational estimates (see Gänsicke et al. 2002) but below theoretical predictions: $10^{-4} \mathrm{pc}^{-3}$ (de Kool 1992) to $2 \times 10^{-5} \mathrm{pc}^{-3}$ (Politano 1996). As we will discuss in Sect. 9.4, the presently known sample of PCEBs/pre-CVs is very likely incomplete not only with respect to those systems containing cold white dwarfs, but also to those systems containing an early-type secondary. Hence, we would like to stress that all the space densities derived above should be regarded as lower limits to the true values.

Considering the binary age postulate (BAP) of King \& Schenker (2002) outlined in Sect. 2 we note that assuming reduced magnetic braking indeed leads to an increase of the averaged evolution time scale for pre-CVs but the presently known
PCEB population is too small and too strongly biased to prove or disprove the BAP scenario.

\section{Observational selection effects}

We have shown that the known population of PCEBs is dominated by young hot systems (Sect. 5.1), and that, applying the current theories of (pre-)CV evolution, the majority of them will remain in a detached configuration for many $10^{8} \mathrm{yrs}$ to several $10^{9}$ yrs (Sect. 6) - sufficiently long for their white dwarf primaries to cool - depending on $t_{\mathrm{sd}}$ and $M_{1}$ - to temperatures $6000 \mathrm{~K} \lesssim T_{\text {eff }} \lesssim 15000 \mathrm{~K}$ (see Fig. 7) These findings strongly suggest that - if the reality about AML in PCEBs is somewhere bracketed between the prescriptions of $\dot{J}_{\mathrm{CMB}}$ and $\dot{J}_{\mathrm{RMB}}-$ the currently known population of PCEBs is highly incomplete.

As a test for possible selection effect, we compare the effective temperature distribution of the white dwarfs in our PCEB sample to that of field white dwarfs.

\subsection{The known PCEB population vs. the McCook \& Sion (1999) white dwarf population}

McCook \& Sion (1999) present a catalogue of 1793 spectroscopically identified white dwarfs, including information on the white dwarf temperatures where available. The temperature distribution of the McCook \& Sion white dwarf sample peaks at temperatures $T_{\text {eff }} \mathrm{WD} \simeq 15000-22000 \mathrm{~K}$ (top panel of Fig. 10) with a mean effective temperature of $\bar{T}_{\text {eff }} \mathrm{wD}=19360 \mathrm{~K}$. Also shown in Fig. 10 (bottom panel) is the temperature distribution of the PCEB white dwarf primaries from our sample (Table 1), which also peaks in the range $T_{\text {eff PCEB }} \simeq 15000-22000 \mathrm{~K}$, but lacks the low-temperature tail seen in the McCook \& Sion sample. The average temperature of the white dwarfs in our 
PCEB sample is $\bar{T}_{\text {eff PCEB }}=33423 \mathrm{~K}$. Using $\chi^{2}$ statistics, the significance that two distributions are different is

$P\left(\frac{v}{2}, \frac{\chi^{2}}{2}\right)=\frac{\int_{0}^{\chi^{2} / 2} \mathrm{e}^{-t} t^{\nu / 2-1} \mathrm{~d} t}{\int_{0}^{\infty} t^{\nu / 2-1} \mathrm{e}^{-t} \mathrm{~d} t}$,

where $v$ is the number of degrees of freedom, i.e. the number of bins, and $\chi^{2}$ is given by:

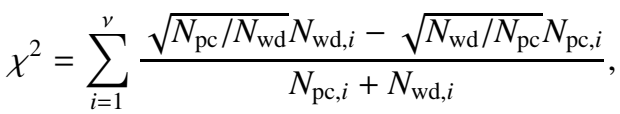

with $N_{i}$ the number of objects in bin $i, N_{\mathrm{wd}} \equiv \sum_{i=1}^{v} N_{\mathrm{wd}, \mathrm{i}}$ and $N_{\mathrm{pc}} \equiv \sum_{i=1}^{v} N_{\mathrm{pc}, \mathrm{i}}$. We obtain for the probability that the two distributions are different:

$P\left(\frac{v}{2}, \frac{\chi^{2}}{2}\right)=0.93$.

It appears, hence, likely that the PCEB white dwarfs are indeed on average hotter - and therefore younger - than the single field white dwarfs of (McCook \& Sion 1999).

\subsection{PCEB and field white dwarfs in the Palomar Green survey}

Whereas the McCook \& Sion (1999) is a heterogenous catalogue of white dwarfs identified by various means, it is of fundamental interest to compare the properties of white dwarfs in PCEBs and of field white dwarfs drawn from a single survey. Inspection of Table 1 shows that five of our PCEBs with a white dwarf primary are contained in the Palomar-Green (PG) survey (Green et al. 1986): UZ Sex, CC Cet, LM Com, GK Vir and NN Ser. Figure 10 shows the temperature distribution of both the field white dwarfs (upper panel) and of the PCEB white dwarfs (bottom panel) from the PG survey. The mean values of the effective temperatures for PCEB white dwarfs and single white dwarfs contained in the PG survey are $\bar{T}_{\text {eff PCEB }}=35380 \mathrm{~K}$ and $\bar{T}_{\text {eff }} \mathrm{wD} \sim 21000 \mathrm{~K}$, respectively. The mean effective temperature of the white dwarfs in PCEBs is again somewhat higher than that of their field relatives. As there are only five PCEBs with a white dwarf primary included in the PG-survey, the statistic is very poor but for completeness we give the probability that the difference of the two distributions (see the shaded histograms in Fig. 10) can be explained by chance

$1-P\left(\frac{v}{2}, \frac{\chi^{2}}{2}\right)=0.51$

Obviously, from this subset of systems we can neither say that the white dwarfs in PCEBs are systematically hotter and younger than their field relatives nor can we exclude that this is the case. In the next section we will discuss observational selection effects. This will give us a hint which possibility is more likely.

Notice, the probabilities given by Eqs. (17) and (18) are calculated by neglecting the very few detected extremely old single white dwarfs, i.e. using only 9 (Eq. (17)) respectively 5 (Eq. (18)) bins.

\subsection{Finding PCEBs}

Based on the presently available data (Sects. 9.1 and 9.2), we can not exclude that the white dwarfs in PCEBs are - on average - systematically hotter (and younger) than single white dwarfs. This statement may be entirely based on selection effects skewing the temperature/age distribution of the white dwarfs in the known PCEB population. It is, however, very difficult to assess this bias quantitatively. We list in Appendix A for the PCEBs from our sample in Table 1 (1) the way that they were discovered in the first place, and (2) the way that their binarity has been identified. Inspection of these notes shows that the vast majority of the known PCEBs have been identified in the first place because of their white dwarf component either as blue object or X-ray source. Obviously, the presence of a late-type companion may significantly affect the colour of a PCEB: the cooler the white dwarf and/or the earlier the companion are, the less blue the system will appear. This may affect different candidate samples in different ways, depending on how the criterion blue has been defined.

We have computed the colours expected for PCEBs containing a white dwarf with $6000 \mathrm{~K}<T_{\text {eff }}<50000 \mathrm{~K}$ and an ZAMS companion with spectral type K0 to M6, the results for $U-B$ and $B-V$ are shown in Fig. 11. Comparing these simulated colours to the selection criterion of the PG survey, $U-B<0.46$ (Green et al. 1986), it becomes apparent that PCEBs containing a cool $\left(T_{\text {eff }} \lesssim 15000 \mathrm{~K}\right)$ white dwarf will be included in the PG survey only if their companions are of spectral type $\sim$ M4 or later. Similarly, for such PCEBs $B-V>0.5$, which will most likely prevent their selection as white dwarf candidates. As an example for the "historic" white dwarf searches, Giclas et al. (1965) used a Lowell colour class -1 or 0 (corresponding to $B-V<0.0$ or $B-V<+0.2$, and $U-B<-0.78$ or $U-B<-0.60)$ as selection criterion. Consequently, the GD lists will contain only those PCEBs containing moderately hot white dwarfs and late type secondaries, such as HR Cam and MS Peg.

PCEBs which were not initially selected as white dwarf candidates are: (1) the planetary nebulae; (2) high proper motion objects (RR Cae, BPM 6502, and BPM 71214) - interestingly enough, RR Cae is the PCEB containing the coldest white dwarf in our sample; and (3) variable stars (V471 Tau: spectroscopic; BE UMa: photometric). In the case of V471 Tau the optical emission is largely dominated by the $\mathrm{K} 2 \mathrm{~V}$ companion, and with $B-V \simeq 0.76$ the system does clearly not qualify as a white dwarf candidate.

\subsection{The lack of early companions}

So far we have focussed much of our attention on the temperatures of the white dwarfs in our PCEB sample, as this parameter is a direct measure of the age of these systems. However, Table 1 suggests that this sample is also biased with respect to the mass of the companion stars. The average mass of the companion in our PCEB sample is $\bar{M}_{\text {sec PCEB }}=0.24$, whereas CVcat (Kube et al. 2002) lists the masses for $80 \mathrm{CVs}$ with $\bar{M}_{\text {sec CV }}=0.43$. A direct consequence of this dominance of low mass companion stars in the known PCEB sample is that 

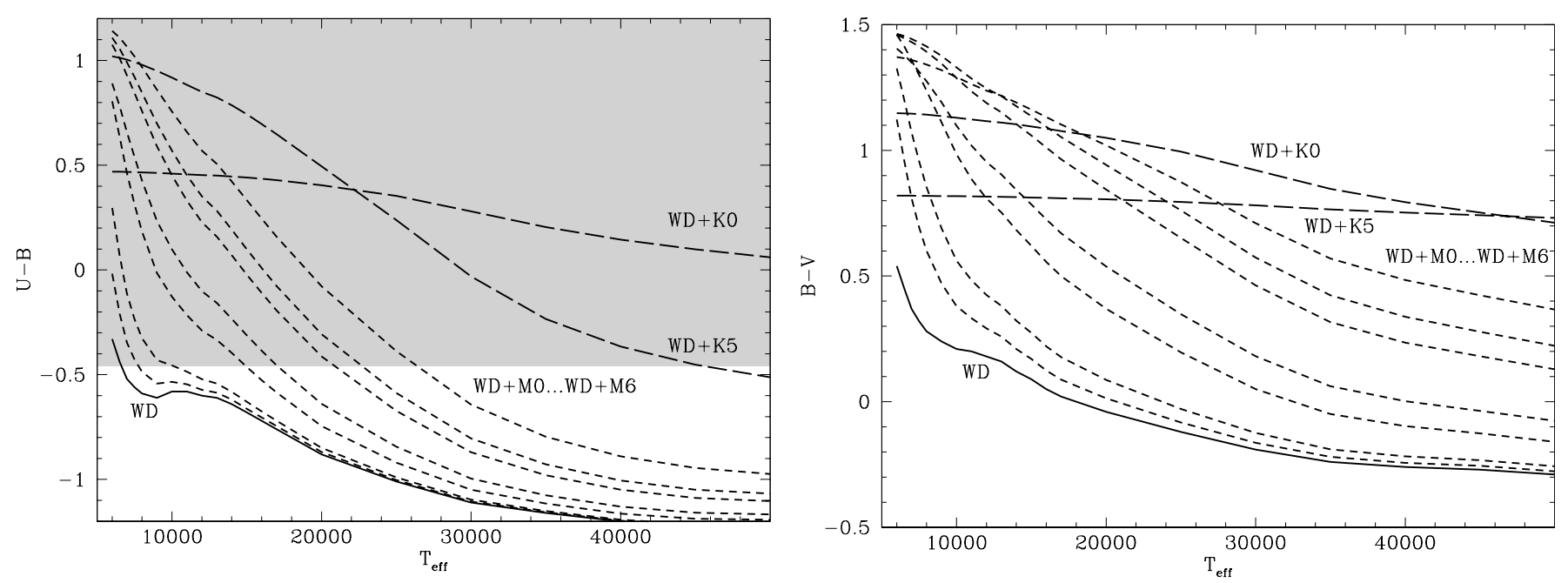

Fig. 11. Simulated colours for PCEBs containing a white dwarf with $6000 \mathrm{~K}<T_{\text {eff }}<50000$ and a companion with a spectral type in the range K0 to M6. The left panel shows in gray the PCEB parameter space that $d o$ not fulfill the selection criterion of the PG survey $(U-B<0.46)$.

the majority of these systems evolve into contact at short orbital periods (Sect. 6, Fig. 5).

The shortage of PCEBs with massive/early-type donor stars $\left(0.4 M_{\odot} \lesssim M_{\text {sec }} \lesssim 0.8 M_{\odot}\right)$, i.e. the progenitors of longperiod CVs, is subject to the selection effect which we just discussed in Sect. 9.3: in PCEBs with an early-type companion the secondary contributes significantly to the total optical emission of the system, and, consequently, such binaries do not qualify as white dwarf candidates (Fig. 11), the major discovery channel for PCEBs.

As a result of the (very likely) incompleteness of known PCEBs with an early-type donor the space density estimates presented in Sect. 8 should be considered to be lower limits to the true $\rho_{\text {PCEB }}$ and $\rho_{\text {preCV }}$.

\section{Conclusion}

We have presented the properties of the currently known sample of well-observed PCEBs and have calculated their past and future evolution based on two different assumptions for the angular momentum loss mechanism, adopting either the "classical" disrupted magnetic braking prescription $\left(\dot{J}_{\mathrm{CMB}}\right)$ or the AML prescription derived by Sills et al. (2000) $\left(\dot{J}_{\mathrm{RMB}}\right)$. The results from this study are:

1. The presently known PCEB population is dominated by young systems most of which have evolved only through a small fraction of their lifetime as detached binaries. On average, the white dwarfs in PCEBs are hotter than field white dwarfs.

2. While the evolution of an individual system strongly depends on whether we assume $\dot{J}_{\mathrm{RMB}}$ or $\dot{J}_{\mathrm{CMB}}$, the total number of pre-CVs within the PCEB sample, i.e. systems which will start mass transfer within a Hubble time, is nearly the same for both prescriptions. Considering the time scale on which the PCEBs will evolve into semi-detached CVs we predict the existence of a large population of old PCEBs containing cold white dwarfs. The present lack of such systems is very likely the result of observational biases, as the majority of the known PCEBs were initially selected as blue objects.

3. An additional consequence of the observational selection effects involved in the discovery of PCEBs is a shortage of systems containing a "massive"/early-type companion $\left(0.4 M_{\odot} \lesssim M_{\mathrm{sec}} \lesssim 0.8 M_{\odot}\right)-$ which are the progenitors of long-period CVs. Indeed, our calculations predict that most of the pre-CVs among the PCEBs in our sample will evolve into $\mathrm{CVs}$ with short orbital periods $\left(P_{\text {orb }}<3 \mathrm{~h}\right)$.

4. The space density of PCEBs estimated from the currently known sample is $6 \times 10^{-6} \mathrm{pc}^{-3} \lesssim \rho_{\text {PCEB }} \lesssim 3 \times 10^{-5} \mathrm{pc}^{-3}$, depending on the assumed AML prescription. Taking into account the age as well as the evolution time scale of the pre-CVs in our sample and assuming that the birthrate of PCEBs remained unchanged we derive for the $\mathrm{CV}$ space density $\rho_{\mathrm{CV}} \sim 10^{-5} \mathrm{pc}^{-3}$, nearly independent on whether we assume $\dot{J}_{\mathrm{CMB}}$ or $\dot{J}_{\mathrm{RMB}}$. This value is somewhat higher than current observational estimates but below theoretical predictions. However, due to the observational bias working against the discovery of PCEBs containing an earlytype companion our estimates should be considered to be lower limits to the true values of $\rho_{\mathrm{CV}}$ and $\rho_{\mathrm{PCEB}}$.

Acknowledgements. We thank for support by an individual MarieCurie Fellowship (MRS) and a PPARC Advanced Fellowship (BTG).

Note added in proof: After the submission of our paper, Raymond et al. (2003) have published a list of 109 PCEBs found in the Sloan Digital Sky Survey. The average white dwarf temperature of this sample $(16000 \mathrm{~K})$ is significantly lower than that of our sample of PCEBs with known orbital period (Table 1), and demonstrates that more sophisticated selection criteria (compared to pure "blue" surveys) can successfully identify PCEBs containing rather cold white dwarfs. Unfortunately, only a single system in the list of Raymond et al. (2003) has an orbital period measurement - determining the periods of their PCEBs harbouring white dwarfs with 
$T_{\text {eff }} \lesssim 12000 \mathrm{~K}$ provides a direct test for our prediction (2) above.

\section{Appendix A: The discovery history of our PCEB sample (Table 1)}

RR Cae: Discovered as high proper motion object by Luyten (1955), DA white dwarf plus Balmer emission line spectrum noted by Rodgers \& Eggen (1974), late-type nature for the companion suggested by Bessell \& Wickramasinghe (1979), discovery of eclipses and orbital period measurement (photometric) by Krzeminski (1984). Two comprehensive studies by Bruch \& Diaz (1998) and Bruch (1999).

EGUMa: Discovered as a white dwarf on Schmidt prism plates by Stephenson (1960), emission lines noted by Greenstein (1965), orbital period (spectroscopic) by Lanning (1982). Recent comprehensive study by Bleach et al. (2000).

EC 13471-1258: Discovered in the Edinburgh-Cape faint blue object survey of high galactic latitudes, binarity revealed through the detection of eclipses (Kilkenny et al. 1997). Recent study by Kawka et al. (2002).

BPM 71214: Discovered as a high proper motion object (Luyten 1963). Recent study by Kawka et al. (2002).

HR Cam: Listed as WD candidate by Giclas et al. (1970), spectroscopic confirmation of WD nature by Wills \& Wills (1974), detection of a red companion by Zuckerman \& Becklin (1992), orbital period (spectroscopic and photometric) from the comprehensive studies of Marsh \& Duck (1996); Maxted et al. (1998).

UZ Sex: Discovered in the PG survey, listed as a DA/composite spectral type by Green et al. (1986), orbital period (spectroscopic) from the comprehensive study of Saffer et al. (1993), see also the recent analyses by Bruch \& Diaz (1999); Bleach et al. (2000).

BPM 6502: Discovered as a high proper motion star by Luyten (1957a), listed as WD candidate by Eggen (1969), spectroscopically confirmed as a WD by Wegner (1973), detection of companion and orbital period (spectroscopic) by Kawka et al. (2000).

HZ 9: Discovered in a search for faint blue stars by Humason \& Zwicky (1947), mentioned as a DA+dMe candidate by Greenstein (1958), orbital period (spectroscopic) measured by Lanning \& Pesch (1981).

MS Peg: Listed as WD candidate by Giclas et al. (1965), spectroscopic confirmation of WD nature by Greenstein (1969), emission lines and radial velocity variations detected by Tytler \& Rubenstein (1989) and Schultz et al. (1993), orbital period (spectroscopic) measured by Schmidt et al. (1995).

HS 0705+6700: Classified in the Hamburg Quasar Survey (Hagen et al. 1995) as hot star candidate, eclipses discovered and orbital period measured by Drechsel et al. (2001).

HW Vir: Listed as ultraviolet-bright star by Carnochan \& Wilson (1983) and classified as likely sdB star by Berger \& Fringant (1980), discovery of eclipses and orbital period (photometric) by Menzies (1986).

LM Com: Listed as blue object by Iriarte \& Chavira (1957), also detected in the PG survey (Green et al. 1986), and identified as DA+dM binary by Ferguson et al. (1984), $\mathrm{H} \alpha$ emission detected by Orosz et al. (1997), orbital period (spectroscopic) and comprehensive study by Orosz et al. (1999).

PG 1017-086: Discovered in the PG survey, classified as sdB star by Green et al. (1986), variability discovered and orbital period measured by Maxted et al. (2002).

CC Cet: Discovered in the PG survey, listed as a DA/composite spectral type by Green et al. (1986), orbital period (spectroscopic) from the comprehensive study of Saffer et al. (1993), see also Somers et al. (1996).

V471 Tau: The brightest and best-studied pre-CV. Listed as a spectroscopic binary by Wilson (1953), discovery of eclipses, spectral classification as DA $+\mathrm{dK}$ binary, and measurement of the orbital period (photometric) by Nelson \& Young (1970).

PG 1336-018: Discovered in the PG survey, classified as sdB star by Green et al. (1986), eclipses discovered and orbital period measured by Kilkenny et al. (1998).

AA Dor: Listed as faint blue star by Luyten (1957b) and as spectroscopically variable object in the foreground of the LMC by Feast et al. (1960), discovery of eclipses and measurement of the orbital period (photometric) by Kilkenny et al. (1978).

RE 2013+400: Discovered as bright EUV source during the ROSAT Wide Field Camera all-sky survey and listed as a WD by Pounds et al. (1993), variable Balmer emission was noted by Barstow et al. (1993), the orbital period (spectroscopic) was measured by Thorstensen et al. (1994).

GK Vir: Discovered in the PG survey, eclipse discovered and orbital period (photometric) measured by Green et al. (1978).

MT Ser: Classified as PN on the Palomar Sky Survey plates by Abell (1955), variability suggested by Abell (1966), recurrent photometric variability was detected by Grauer \& Bond (1983) who also suggested the sdO+dM nature of the system, for a more comprehensive photometric study, see Bruch et al. (2001).

IM CMa: Discovered as bright EUV source during the ROSAT Wide Field Camera all-sky survey and listed as a WD by Pounds et al. (1993), Balmer emission detected and orbital period (spectroscopic) measured by Vennes \& Thorstensen (1994).

NN Ser: Discovered as a CV candidate in the PG survey by Green et al. (1986), discovery of deep eclipses, classification as $\mathrm{DA}+\mathrm{dM}$ binary and measurement of the orbital period (photometric) by Haefner (1989).

RE 1016-053: Discovered as bright EUV source during the ROSAT Wide Field Camera all-sky survey and listed as a WD by Pounds et al. (1993), classified as DA+dM by Jomaron et al. (1993), orbital period (spectroscopic) measured by Tweedy et al. (1993).

V477Lyr: Classified as PN on the Palomar Sky Survey plates by Abell (1955), variability suggested by Abell (1966), eclipse discovered and period (photometric) measured by Bond (1980).

Abell 65: Classified as PN on the Palomar Sky Survey plates by Abell (1966), an estimate of the orbital period (photometric) was published by Bond \& Livio (1990).

KV Vel: Listed as luminous star in the list of Stephenson \& Sanduleak (1971), planetary nebula nature suggested by Holmberg et al. (1978), spectroscopically identified as sdO star 
within a planetary nebula by Drilling (1983). Photometric variability discovered and orbital period (photometric) measured by Drilling \& Bravo (1984).

UUSge: Classified as PN on the Palomar Sky Survey plates by Abell (1955), variability suggested by Abell (1966), eclipses discovered and orbital period (photometric) measured by Miller et al. (1976).

HS 1136+6646: Classified in the Hamburg Quasar Survey (Hagen et al. 1995) as hot star candidate, identified as a spectroscopic binary by Heber et al. (1996), orbital period (spectroscopic) measured by Sing et al. (2001).

BE UMa: Identified as variable star and orbital period (photometric) by Kurochkin $(1964,1971)$, recovered as emission line star in the PG survey by Ferguson et al. (1981).

\section{References}

Abell, G. O. 1955, PASP, 67, 258

Abell, G. O. 1966, ApJ, 144, 259

Althaus, L. G., \& Benvenuto, O. G. 1997, ApJ, 477, 313

Andronov, N., Pinsonneault, M., \& Sills, A. 2003, ApJ, 582, 358

Barstow, M. A., Hodgkin, S. T., Pye, J. P., et al. 1993, in White Dwarfs: Advances in Observation and Theory, ed. M. A. Barstow, NATO ASIC Proc. No. 403 (Dordrecht: Kluwer), 433

Bell, S. A., Pollacco, D. L., \& Hilditch, R. W. 1994, MNRAS, 270, 449

Berger, J., \& Fringant, A.-M. 1980, A\&A, 85, 367

Bergeron, P., Wesemael, F., Beauchamp, A., et al. 1994, ApJ, 432, 305

Bessell, M. S., \& Wickramasinghe, D. T. 1979, ApJ, 227, 232

Bleach, J. N., Wood, J. H., Catalán, M. S., et al. 2000, MNRAS, 312, 70

Bond, H. E. 1980, IAU Circ., 3480

Bond, H. E., \& Livio, M. 1990, ApJ, 355, 568

Bragaglia, A., Renzini, A., \& Bergeron, P. 1995, ApJ, 443, 735

Bruch, A. 1999, AJ, 117, 3031

Bruch, A., \& Diaz, M. P. 1998, AJ, 116, 908

Bruch, A., \& Diaz, M. P. 1999, A\&A, 351, 573

Bruch, A., Vaz, L. P. R., \& Diaz, M. P. 2001, A\&A, 377, 898

Cahn, J. H., \& Kaler, J. B. 1971, ApJS, 22, 319

Cahn, J. H., Kaler, J. B., \& Stanghellini, L. 1992, A\&AS, 94, 399

Carnochan, D. J., \& Wilson, R. 1983, MNRAS, 202, 317

Catalan, M. S., Davey, S. C., Sarna, M. J., Connon-Smith, R., \& Wood, J. H. 1994, MNRAS, 269, 879

Cayrel, R., Hill, V., Beers, T. C., et al. 2001, Nature, 409, 691

Chen, A., O'Donoghue, D., Stobie, R. S., et al. 1995, MNRAS, 275 , 100

Clemens, J. C., Reid, I. N., Gizis, J. E., \& O’Brien, M. S. 1998, ApJ, 496, 352

de Kool, M. 1992, A\&A, 261, 188

de Kool, M., \& Ritter, H. 1993, A\&A, 267, 397

Downes, R. A. 1986, ApJ, 307, 170

Drechsel, H., Heber, U., Napiwotzki, R., et al. 2001, A\&A, 379, 893

Driebe, T., Blöcker, T., Schönberner, D., \& Herwig, F. 1999, A\&A, 350, 89

Driebe, T., Schoenberner, D., Bloecker, T., \& Herwig, F. 1998, A\&A, 339, 123

Drilling, J. S. 1983, ApJ, 270, L13

Drilling, J. S., \& Bravo, J. 1984, IAU Circ., 3939

Dubus, G., Taam, R. E., \& Spruit, H. C. 2002, ApJ, 569, 395

Eggen, O. J. 1969, ApJ, 157, 287

Eggleton, P. P. 1983, ApJ, 268, 368
Feast, M. W., Thackeray, A. D., \& Wesselink, A. J. 1960, MNRAS, 121,337

Ferguson, D. H., Green, R. F., \& Liebert, J. 1984, ApJ, 287, 320

Ferguson, D. H., Liebert, J., Haas, S., Napiwotzki, R., \& James, T. A. 1999, ApJ, 518, 866

Ferguson, D. H., McGraw, J. T., Spinrad, H., Liebert, J., \& Green, R. F. 1981, ApJ, 251, 205

Ferreras, I., Melchiorri, A., \& Silk, J. 2001, MNRAS, 327, L47

Fontaine, G., Brassard, P., \& Bergeron, P. 2001, PASP, 113, 409

Fujimoto, M. Y., \& Iben, I. J. 1989, ApJ, 341, 306

Fulbright, M. S., Liebert, J., Bergeron, P., \& Green, R. 1993, ApJ, 406, 240

Gänsicke, B. T. 2000, Reviews of Modern Astronomy, 13, 151

Gänsicke, B. T., Beuermann, K., \& de Martino, D. 1995, A\&A, 303, 127

Gänsicke, B. T., Hagen, H. J., \& Engels, D. 2002, On the space density of cataclysmic variables, ed. B. T. Gänsicke, K. Beuermann, \& K. Reinsch, ASP Conf. Ser., 261, 190

Giclas, H. L., Burnham, R., \& Thomas, N. G. 1965, Lowell Obs. Bull., 125,155

Giclas, H. L., Burnham, R., \& Thomas, N. G. 1970, Lowell Obs. Bull., 153,183

Grauer, A. D., \& Bond, H. E. 1983, ApJ, 271, 259

Green, R. F., Liebert, J., \& Wesemael, F. 1984, ApJ, 280, 177

Green, R. F., Richstone, D. O., \& Schmidt, M. 1978, ApJ, 224, 892

Green, R. F., Schmidt, M., \& Liebert, J. 1986, ApJS, 61, 305

Greenstein, J. L. 1958, Handbuch der Physik, vol. 50 (Berlin: Springer), 161

Greenstein, J. L. 1965, in First Conference on Faint Blue Stars, ed. W. Luyten (Minneapolis: University of Minnesota Press), 97

Greenstein, J. L. 1969, ApJ, 158, 281

Guinan, E. F., \& Sion, E. M. 1984, AJ, 89, 1252

Haefner, R. 1989, A\&A, 213, L15

Hagen, H. J., Groote, D., Engels, D., \& Reimers, D. 1995, A\&AS, 111,195

Heber, U., Dreizler, S., \& Hagen, H.-J. 1996, A\&A, 311, L17

Herrero, A., Manchado, A., \& Mendez, R. H. 1990, Ap\&SS, 169, 183

Hilditch, R. W., Harries, T. J., \& Hill, G. 1996, MNRAS, 279, 1380

Holmberg, E. B., Lauberts, A., Schuster, H. E., \& West, R. M. 1978, A\&AS, 31, 15

Howell, S. B., Nelson, L. A., \& Rappaport, S. 2001, ApJ, 550, 897

Howell, S. B., Rappaport, S., \& Politano, M. 1997, MNRAS, 287, 929

Humason, M. L., \& Zwicky, F. 1947, ApJ, 105, 85

Iben, I. J., \& Tutukov, A. V. 1993, ApJ, 418, 343

Iriarte, B., \& Chavira, E. 1957, Boletin de los Observatorios Tonantzintla y Tacubaya, 2, 3

Jomaron, C. M., Raymont, G. B., Bromage, G. E., et al. 1993, MNRAS, 264, 219

Kawka, A., Vennes, S., Dupuis, J., \& Koch, R. 2000, AJ, 120, 3250

Kawka, A., Vennes, S., Koch, R., \& Williams, A. 2002, AJ, 124, 2853

Kepler, S. O., \& Nelan, E. P. 1993, AJ, 105, 608

Kilkenny, D., Hilditch, R. W., \& Penfold, J. E. 1978, MNRAS, 183 , 523

Kilkenny, D., O’Donoghue, D., Koen, C., Lynas-Gray, A. E., \& van Wyk, F. 1998, MNRAS, 296, 329

Kilkenny, D., O'Donoghue, D., Koen, C., Stobie, R. S., \& Chen, A. 1997, MNRAS, 287, 867

King, A. R. 1988, QJRAS, 29, 1

King, A. R., Frank, J., Kolb, U., \& Ritter, H. 1995, ApJ, 444, L37

King, A. R., \& Kolb, U. 1995, ApJ, 439, 330

King, A. R., Kolb, U., De Kool, M., \& Ritter, H. 1994, MNRAS, 269, 907

King, A. R., Osborne, J. P., \& Schenker, K. 2002, MNRAS, 329, L43 
King, A. R., \& Schenker, K. 2002, in The Physics of Cataclysmic Variables and Related Objects, ed. B. T. Gänsicke, K. Beuermann, \& K. Reinsch, ASP Conf. Ser., 261, 233

Koester, D. 2002, A\&AR, 11, 33

Koester, D., Schulz, H., \& Weidemann, V. 1979, A\&A, 76, 262

Kolb, U. 2002, in The Physics of Cataclysmic Variables and Related Objects, ed. B. T. Gänsicke, K. Beuermann, \& K. Reinsch, ASP Conf. Ser., 261, 180

Kolb, U., \& Baraffe, I. 1999, MNRAS, 309, 1034

Kolb, U., King, A. R., \& Ritter, H. 1998, MNRAS, 298, L29

Krishnamurthi, A., Pinsonneault, M. H., Barnes, S., \& Sofia, S. 1997, ApJ, 480, 303

Krzeminski, W. 1984, IAU Circ., 4014

Kube, J., Gänsicke, B., \& Hoffmann, B. 2002, in The Physics of Cataclysmic Variables and Related Objects, ed. B. T. Gänsicke, K. Beuermann, \& K. Reinsch, ASP Conf. Ser., 261, 678

Kudritzki, R. P., Simon, K. P., Lynas-Gray, A. E., Kilkenny, D., \& Hill, P. W. 1982, A\&A, 106, 254

Kurochkin, N. E. 1964, Peremnye Zvezdy (Academy Sci. U.S.S.R., Var. Star Bull), 15, 77

Kurochkin, N. E. 1971, Peremnye Zvezdy (Academy Sci. U.S.S.R., Var. Star Bull), 18, 85

Lanning, H. H. 1982, ApJ, 253, 752

Lanning, H. H., \& Pesch, P. 1981, ApJ, 244, 280

Livio, M., \& Pringle, J. E. 1994, ApJ, 427, 956

Luyten, W. J. 1955, Luyten's Five Tenths (Minneapolis: Lund Press)

Luyten, W. J. 1957a, A catalogue of 9867 stars in the Southern Hemisphere with proper motions exceeding $0 ! 2$ annually (Minneapolis: Lund Press)

Luyten, W. J. 1957b, A Search for Faint Blue Stars (Minneapolis: University of Minnesota)

Luyten, W. J. 1963, Bruce proper motion survey. The general catalogue, Vol. I, II

Maciel, W. J. 1984, A\&AS, 55, 253

Marsh, T. R., Dhillon, V. S., \& Duck, S. R. 1995, MNRAS, 275, 828

Marsh, T. R., \& Duck, S. R. 1996, MNRAS, 278, 565

Maxted, P. F. L., Marsh, T. R., Heber, U., et al. 2002, MNRAS, 333, 231

Maxted, P. F. L., Marsh, T. R., Moran, C., Dhillon, V. S., \& Hilditch, R. W. 1998, MNRAS, 300, 1225

McCook, G. P., \& Sion, E. M. 1999, ApJS, 121, 1

Menzies, J. W. 1986, Ann. Rep. SAAO, 1985, 20

Miller, J. S., Krzeminski, W., \& Priedhorsky, W. 1976, IAU Circ., 2974

Nelson, B., \& Young, A. 1970, PASP, 82, 699

O’Brien, M. S., Bond, H. E., \& Sion, E. M. 2001, ApJ, 563, 971

Orosz, J., Wade, R. A., \& Harlow, J. J. B. 1997, AJ, 114, 317

Orosz, J. A., Wade, R. A., Harlow, J. J. B., et al. 1999, AJ, 117, 1598

Paczynski, B., \& Sienkiewicz, R. 1983, ApJ, 268, 825

Patterson, J. 1984, ApJS, 54, 443

Patterson, J. 1998, PASP, 110, 1132

Perryman, M. A. C., Brown, A. G. A., Lebreton, Y., et al. 1998, A\&A, 331,81

Pinsonneault, M. H., Andronov, N., \& Sills, A. 2002, in The Physics of Cataclysmic Variables and Related Objects, ed. B. T. Gänsicke, K. Beuermann, \& K. Reinsch, ASP Conf. Ser., 261, 208

Politano, M. 1996, ApJ, 465, 338

Pollacco, D. L., \& Bell, S. A. 1993, MNRAS, 262, 377

Pollacco, D. L., \& Bell, S. A. 1994, MNRAS, 267, 452
Pottasch, S. R. 1996, A\&A, 307, 561

Pounds, K. A., Allan, D. J., Barber, C., et al. 1993, MNRAS, 260, 77 Prialnik, D., \& Livio, M. 1985, MNRAS, 216, 37

Rappaport, S., Joss, P. C., \& Verbunt, F. 1983, ApJ, 275, 713

Rauch, T. 2000, A\&A, 356, 665

Raymond, S. N., Szkody, P., Hawley, S. L., et al. 2003, AJ, 125, 2621

Ringwald, F. A. 1996, in Cataclysmic Variables and Related Objects, ed. A. Evans, \& J. H. Wood, IAU Coll. No. 158 (Dordrecht: Kluwer), 89

Ritter, H. 1986, A\&A, 169, 139

Ritter, H., \& Kolb, U. 1998, A\&AS, 129, 83

Rodgers, A. W., \& Eggen, O. J. 1974, PASP, 86, 742

Saffer, R. A., Wade, R. A., Liebert, J., et al. 1993, AJ, 105, 1945

Schenker, K., \& King, A. R. 2002, in The Physics of Cataclysmic Variables and Related Objects, ed. B. T. Gänsicke, K. Beuermann, \& K. Reinsch, ASP Conf. Ser., 261, 242

Schmidt, G. D., Smith, P. S., Harvey, D. A., \& Grauer, A. D. 1995, AJ, 110, 398

Schultz, G., Zuckerman, B., Becklin, E. E., \& Barnbaum, C. 1993, BAAS, 25, 824

Sills, A., Pinsonneault, M. H., \& Terndrup, D. M. 2000, ApJ, 534, 335

Sing, D. K., Holberg, J. B., Howell, S., Barstow, M. A., \& Burleigh, M. 2001, American Astronomical Society Meeting, 199

Somers, M. W., Lockley, J. J., Naylor, T., \& Wood, J. H. 1996, MNRAS, 280, 1277

Spruit, H. C., \& Ritter, H. 1983, A\&A, 124, 267

Spruit, H. C., \& Taam, R. E. 2001, ApJ, 548, 900

Stauffer, J. R. 1987, AJ, 94, 996

Stephenson, C. B. 1960, PASP, 72, 387

Stephenson, C. B., \& Sanduleak, N. 1971, Luminous stars in the Southern Milky Way, Vol. 1 (Publications of the Warner \& Swasey Observatory)

Thorstensen, J. R., Vennes, S., \& Shambrook, A. 1994, AJ, 108, 1924

Townsley, D. M., \& Bildsten, L. 2002, in The Physics of Cataclysmic Variables and Related Objects, ed. B. T. Gänsicke, K. Beuermann, \& K. Reinsch, ASP Conf. Ser., 261, 31

Tweedy, R. W., Holberg, J. B., Barstow, M. A., et al. 1993, AJ, 105, 1938

Tytler, D., \& Rubenstein, E. 1989, in White dwarfs, ed. G. Wegner, IAU Coll. No. 158 (Heidelberg: Springer), 524

Vennes, S., \& Thorstensen, J. R. 1994, ApJ, 433, L29

Vennes, S., \& Thorstensen, J. R. 1996, AJ, 112, 284

Vennes, S., Thorstensen, J. R., \& Polomski, E. F. 1999, ApJ, 523, 386

Verbunt, F., \& Zwaan, C. 1981, A\&A, 100, L7

Walsh, J. R., \& Walton, N. A. 1996, A\&A, 315, 253

Walton, N. A., Walsh, J. R., \& Pottasch, S. R. 1993, A\&A, 275, 256

Warner, B. 1995, Cataclysmic Variable Stars (Cambridge: Cambridge University Press)

Wegner, G. 1973, MNRAS, 163, 381

Weidemann, V. 2000, A\&A, 363, 647

Wills, D., \& Wills, B. J. 1974, MNRAS, 167, 79P

Wilson, R. E. 1953, General Catalogue of Stellar Radial Velocities (Carnegie Institute Washington D.C. Publication)

Wood, J. H., \& Marsh, T. R. 1991, ApJ, 381, 551

Wood, J. H., Robinson, E. L., \& Zhang, E.-H. 1995, MNRAS, 277, 87

Wood, J. H., \& Saffer, R. 1999, MNRAS, 305, 820

Wood, M. A. 1995, in White Dwarfs, ed. D. Koester, \& K. Werner, LNP No. 443 (Heidelberg: Springer), 41

Zuckerman, B., \& Becklin, E. E. 1992, ApJ, 386, 260 